\title{
Flow and geometrical effects on radiated noise from exhaust pipes computed by the Fourier pseudospectral time-domain method
}

\author{
Maarten Hornikx ${ }^{\mathrm{a}, *}$, Wim De Roeck ${ }^{\mathrm{b}}$, Thomas Toulorge ${ }^{\mathrm{c}}$, Wim Desmet ${ }^{\mathrm{b}}$ \\ ${ }^{a}$ Building Physics and Services, Eindhoven University of Technology, P.O. box 513, 5600 MB \\ Eindhoven, the Netherlands. \\ ${ }^{b}$ Mechanical Engineering, K.U.Leuven, Celestijnenlaan 300 B, B-3001, Leuven, Belgium. \\ ${ }^{c}$ Institute of Mechanics, Materials and Civil engineering, Université catholique de Louvain, Avenue \\ Georges Lemaître 4, 1348 Louvain-la-Neuve, Belgium.
}

\begin{abstract}
The current work initializes a systematic numerical study on the combined effects of the geometry surrounding an automotive exhaust pipe, i.e. the ground surface and the automotive body, as well as the influence of the non-uniform velocity and temperature from the exhaust jet, on the acoustic radiation pattern of the pipe. The problem is treated by first solving the RANS equations to obtain the mean velocity and temperature solution of the exhaust jet. The acoustic radiation is computed by solving the linearized Euler (LEE) equations. For an efficient solution of the LEE, a recently developed multi-domain extended Fourier pseudospectral time-domain (PSTD) methodology is used. This method combines the favorable spectral accuracy of the PSTD method with a local grid refinement in the region with high gradients of the mean flow and temperature fields. A filter was found necessary for numerical stability of the PSTD method for this application. For radiation of noise from an exhaust pipe in free field, results from PSTD show a good agreement with reference results, both for cases with and without a jet flow. Results show that the presence of a rigid ground surface and simplified automotive body is increases the radiated sound power by $6 \mathrm{~dB}$ for the lower frequency region, and the effect of the body on the directivity is
\end{abstract}

\footnotetext{
${ }^{*}$ Corresponding author. Tel.: +31 40 2474236; Fax: +31 402438595 .

Email address: m.c.j.hornikx@tue.nl (Maarten Hornikx)
} 
largest for the higher frequencies. Flow effects slightly increase the shielding effect of the body for all frequencies, but have a main impact behind the exhaust pipe, where low frequencies experience higher levels and a cone of low sound levels characterizes the high frequencies.

Keywords: linearized Euler equations, pseudospectral time-domain method, tailpipe noise, flow acoustics

\section{Introduction}

As an estimated number of 210 million European people (44\% of the population of the $27 \mathrm{EU}$ member states except Cyprus and Malta) is regularly exposed to road traffic noise levels exceeding $55 \mathrm{~dB}(\mathrm{~A})$ (the WHO threshold of serious annoyance [1]), reduction of road traffic noise in urban areas is of major concern. From road traffic in urban areas, with typical speeds lower than $50 \mathrm{~km} / \mathrm{h}$, the main noise source is due to the internal combustion engine, see e.g. Jonasson et al.[2]. This noise is radiated by structural vibrations of the vehicle and the intake and exhaust pipes, which is called shell noise. Also, noise is radiated from the orifices of the intake and exhaust pipe systems. This latter source is considered in this paper.

In recent EU-funded projects, outdoor sound propagation models and emission models for European road vehicles have been presented in order to generate the obligatory noise maps of EU member states' larger agglomerations [2,3]. A simple model is constructed describing the sound power from tyre-road and propulsion noise as a function of the vehicle speed and type. In the propagation model, the vehicles are modelled by point sources with two or three source heights, which determine the interference effects with the ground surface. The point sources can be assigned both a horizontal and a vertical directivity [2]. A vertical, source type independent, directivity is determined with the main purpose to take into account car body screening. A horizontal directivity function is proposed for tyre-road noise. For propulsion noise, a directivity is included only for heavy vehicles. All these emission and directivity models are derived from laboratory, test-site and on-site measurements. As a consequence, the 
separate effects that determine the directivity of road traffic noise sources have not been distinguished systematically. Accurate computational results for the directivity of exhaust pipe noise sources are, to the authors' knowledge, non-existing, and could contribute to further improve the directivity functions in use.

The exhaust flow leaving an automotive tailpipe influences the radiated noise. Theoretical work on sound radiation from a semi-infinite pipe without flow has been published by Levine and Schwinger [4] and by Munt $[5,6]$ in the presence of a subsonic jet. These models offer a solution for relatively simple cases and can serve as a reference solution for numerical methods needed to handle more complex geometries and flow conditions. A commonly used numerical approach for sound propagation in a flow is to first compute the mean flow field by solving the Navier-Stokes equations. Then, acoustic propagation is computed by numerically solving the linearized Euler equations (LEE) and, for far field propagation, the Kirchhoff-Helmholtz integral as done in references $[7,8]$ or the approach of Ffowcs-Williams and Hawkings; see e.g. [9]. The LEE include the refraction effects of the mean flow on sound propagation as well as the linear coupling of the acoustic modes with the vorticity and entropy modes. Once excited, the latter two modes might turn unstable due to the absence of non-linear effects and molecular viscosity terms in the LEE. Several ways to avoid the solution from growing unstable have been adopted, see e.g. [10]. A gradually spreading shear layer between the jet and the ambient air is present in the application under consideration. It is expected that the acoustic perturbation at the pipe exit will excite a hydrodynamic instability wave which will turn stable as the shear layer is thick enough. The LEE equations for the sound radiation from a jet pipe can be solved by various numerical methods, e.g. the finite volume method [7], the finite difference method $[9,11]$ and the discontinuous Galerkin (DG) method $[12,13]$. These methods do not inherently make use of the underlying wave phenomena, and typically require 6-10 degrees of freedom to resolve an acoustic wavelength. A more efficient method that candidates to solve the LEE for relatively simple geometries is the Fourier pseudospectral time-domain (PSTD) method [14]. This method has been used before 
in aeroacoustics for unbounded domains or directions, and has recently been applied to Cartesian configurations with rigid boundaries and fluids with piecewise constant densities for a range of outdoor sound propagation applications $[15,16,17]$ and indoor spaces [18]. No instability problems were reported in these studies. The strengths of the PSTD method concern the computational cost and storage requirements, since only two spatial grid points are needed to resolve a wavelength. This is especially of interest for small-wavelength three-dimensional (3-D) applications. Although curved boundaries cannot be resolved by nature using the Fourier PSTD method as it relies on a Cartesian grid, the recent multi-domain PSTD development allows to locally refine the grid, making the method more efficient and applicable to a wider range of geometries [19].

The purpose of this study is to evaluate the performance of the cost-efficient Fourier PSTD method for studying the influence of the jet on the acoustic radiation from an automotive exhaust pipe by solving the LEE. Furthermore, this paper focuses on a numerical study, using the Fourier PSTD method, to the combined effect of surrounding geometry and jet flow on the power and directivity of the radiated sound field from exhaust pipes. The noise originating from the combustion engine is studied, and noise caused by the turbulence behind the tailpipe is not considered here.

This paper is organized as follows. In Section 2, the studied configuration of the exhaust pipe in the presence of a ground surface and an automotive body, as well as the jet flow configurations, are described. The equations that govern sound radiation from an automotive exhaust pipe can be found in Section 3 as well as the outline of the computational approach of solving these equations. Furthermore, the essentials of the utilized multi-domain methodology are briefly addressed in this section. In Section 4, the accuracy of the PSTD method is demonstrated for sound radiation from an axisymmetric exhaust pipe with and without jet by comparing with reference results. Instability issues are addressed, grid convergence is studied and filtering of the mean flow velocity field is discussed. The results from the numerical study are presented in Section 5. Finally, conclusions can be found in Section 6. It needs to 
be stressed that the current study is a preliminary study on the combined effects of flow and surrounding geometry. Plans of continued work are also addressed in this section.

\section{Studied configurations}

Two configurations of sound radiation from an exhaust pipe are considered, see Figs. B.1 and B.2(a). While the first configuration is used for validation purposes, the second configuration is used to study the influence of the flow properties and effects of the geometry surrounding an automotive exhaust pipe on the radiated sound.

\subsection{Validation configuration}

An axisymmetric case of a round pipe in free field has been chosen, as analytical models are available for this configuration. The pipe has a diameter of $D=2 a=40$ $\mathrm{mm}$, being a typical dimension of an automotive exhaust duct. As the first transverse pipe modes start at approximately $5 \mathrm{kHz}$ (for an adiabatic sound speed of $340 \mathrm{~m} / \mathrm{s}$ ), the dominance of the acoustic power in the lower frequency region (see e.g. Torregrosa et al. [20]) motivates the choice of only considering plane incident sound waves. This incident broadband plane sound wave represents sound being generated in the combustion engine. When a jet flow is considered in the tailpipe, an inflow velocity of $u_{0}=104 \mathrm{~m} / \mathrm{s}$, corresponding to a Mach number of $M=u_{0} / c_{0}=0.3$, is used in the pipe. A uniform temperature is assumed.

\subsection{Automotive configuration}

The geometry surrounding an automotive exhaust pipe typically consists of a ground surface and the automotive body. In this initial study, this geometry is simplified in a 3-D configuration: the ground surface is represented by a rigid horizontal plane below the pipe and the automotive body by a rigid cuboid on top of the tailpipe, see Fig. B.2(a). As the solution method of the LEE relies on an equidistant grid, an exhaust pipe with a rectangular cross section is modelled. In particular, the exhaust 
pipe measures $40 \mathrm{~mm}$ x $40 \mathrm{~mm}$ x $4120 \mathrm{~mm}$ in respectively the $z, y$ and $x$-direction and is located $0.28 \mathrm{~m}$ above the ground surface, placed off center in the width of the body and its exit is located at $0.12 \mathrm{~m}$ from the end of the body. The cuboid measures $1.08 \mathrm{~m} \times 1.72 \times 4.00 \mathrm{~m}$ in respectively the $z, y$ and $x$ direction, a volume in accordance with realistic automotive volumes. The automotive body is extremely simplified with the aim to study first order geometrical effects. Computations are made for the configuration of Fig. B.2(a) as well as for the same exhaust duct in the absence of the body and ground surface. For the problem under consideration, an exhaust jet flows through the pipe. The jet has an inflow velocity of $M=0.3$ and has either an ambient temperature of $T=293 \mathrm{~K}$ or an inlet temperature of $T_{0}=723$ $K$, further referred to as the cold and hot jet (Note that the conditions of $u_{0}=104$ $\mathrm{m} / \mathrm{s}$ and $T_{0}=723 \mathrm{~K}$ imply an inlet Mach number of $\left.M=0.19\right)$. These jet properties represent upper values for an automotive exhaust engine, see e.g. reference [21]. Finally, the influence of inflow velocity and inlet temperature is studied for a range of velocities and temperatures representing various automotive exhaust engine speeds. Results are presented in 1/3-octave bands and since exhaust pipe noise has most of its energy in the lower frequency region, i.e. around $100 \mathrm{~Hz}$, the analysis is limited to frequencies of the $2500 \mathrm{~Hz} 1 / 3$-octave band. All frequencies are below the cut-on frequency of transversal duct modes.

\section{Computational approach}

\subsection{Linearized Euler equations (LEE)}

The equations that govern the physical phenomena of noise radiation from the exhaust pipe are the compressible Navier-Stokes (NS) equations. Effects of molecular viscosity are neglected and all physical variables are decomposed into their background values, including convective and thermal effects, denoted by subscript 0 , and acoustic fluctuations:

$$
\rho_{\text {tot }}=\rho_{0}+\rho,
$$




$$
\begin{aligned}
& \mathbf{u}_{\mathrm{tot}}=\mathbf{u}_{0}+\mathbf{u}, \\
& p_{\mathrm{tot}}=p_{0}+p,
\end{aligned}
$$

with $\rho$ the density, $\mathbf{u}=\left[u_{z}, u_{y}, u_{z}\right]^{T}$ the velocity vector and $p$ the pressure. As reported e.g. by Davies and Holland [22], the amplitudes of the acoustic fluctuations in an automotive exhaust pipe may reach the non-linear regime, yet with peak to mean pressure amplitudes up to 1.1. As a result, the error, introduced by linearizing with respect to the acoustic variables, is expected to be small. In this way, the linearized Euler equations (LEE) [14] in non-conservative form are obtained:

$$
\begin{aligned}
& \frac{\partial \mathbf{q}}{\partial t}+\mathbf{A}_{j} \frac{\partial \mathbf{q}}{\partial j}+\mathbf{C q}=\mathbf{q}_{s r}, \\
& \mathbf{A}_{j}=\left[\begin{array}{ccccc}
u_{0, j} & \rho_{0} \delta_{x, j} & \rho_{0} \delta_{y, j} & \rho_{0} \delta_{z, j} & 0 \\
0 & u_{0, j} & 0 & 0 & \frac{\delta_{x, j}}{\rho_{0}} \\
0 & 0 & u_{0, j} & 0 & \frac{\delta_{y, j}}{\rho_{0}} \\
0 & 0 & 0 & u_{0, j} & \frac{\delta_{z, j}}{\rho_{0}} \\
0 & \gamma p_{0} \delta_{x, j} & \gamma p_{0} \delta_{y, j} & \gamma p_{0} \delta_{z, j} & u_{0, j}
\end{array}\right] \\
& \mathbf{C}=\left[\begin{array}{ccccc}
\frac{\partial u_{0, j}}{\partial j} & \frac{\partial \rho_{0}}{\partial x} & \frac{\partial \rho_{0}}{\partial y} & \frac{\partial \rho_{0}}{\partial z} & 0 \\
\frac{u_{0, j}}{\rho_{0}} \frac{\partial u_{0, x}}{\partial j} & \frac{\partial u_{0, x}}{\partial x}+D & \frac{\partial u_{0, x}}{\partial y} & \frac{\partial u_{0, x}}{\partial z} & 0 \\
\frac{u_{0, j}}{\rho_{0}} \frac{\partial u_{0, y}}{\partial j} & \frac{\partial u_{0, y}}{\partial x} & \frac{\partial u_{0, y}}{\partial y}+D & \frac{\partial u_{0, y}}{\partial z} & 0 \\
\frac{u_{0, j}}{\rho_{0}} \frac{\partial u_{0, z}}{\partial j} & \frac{\partial u_{0, z}}{\partial x} & \frac{\partial u_{0, z}}{\partial y} & \frac{\partial u_{0, z}}{\partial z}+D & 0 \\
0 & \frac{\partial p_{0}}{\partial x} & \frac{\partial p_{0}}{\partial y} & \frac{\partial p_{0}}{\partial z} & \gamma \frac{\partial u_{0, j}}{\partial j}
\end{array}\right], \\
& D=\frac{\partial u_{0, j}}{\partial j}+\frac{u_{0, j}}{\rho_{0}} \frac{\partial \rho_{0}}{\partial j},
\end{aligned}
$$

with $\mathbf{q}=\left[\rho, u_{x}, u_{y}, u_{z}, p\right]^{T}, \mathbf{q}_{s r}$ the source vector, index $j$ equals $x, y$ or $z, \gamma$ the heat capacity ratio and $\delta$ the Kronecker delta function. 
The following source vector is used:

$$
\begin{aligned}
& \mathbf{q}_{s r}(\mathbf{x}, t)=\left[\begin{array}{c}
\frac{\delta\left(x-x_{s r}\right)}{c_{0}^{2}} \sin \left(2 \pi f_{s r} t\right) e^{-b\left(t-t_{s r}\right)^{2}} \\
0 \\
0 \\
0 \\
\mathbf{q}_{s r}(\mathbf{x}, t)=0 \\
\delta\left(x-x_{s r}\right) \sin \left(2 \pi f_{s r} t\right) e^{-b\left(t-t_{s r}\right)^{2}}
\end{array}\right] \text { for } \quad y, z<|a|, \\
& \text { for } y, z>|a|,
\end{aligned}
$$

with $a$ the half pipe width, $\mathbf{x}=[x, y, z], x_{s r} \mathrm{~m}$ the source location, $t_{s r}=0.001 \mathrm{~s}$ is the central time, $b=3 f_{s}^{2} / 16$ a constant determining the spectral bandwidth, $\delta\left(x-x_{s r}\right)$ the Dirac delta function. $f_{s r}=f_{s} / 8 \mathrm{~Hz}$ the central frequency with $f_{s}=c_{0} / \Delta x$ the sample frequency corresponding to the spatial discretization. The source conditions are assumed to be adiabatic as the background pressure and density are set constant in the tailpipe. The calculations are accompanied by the following boundary conditions:

$$
\frac{\partial p\left(\mathbf{x}_{\Gamma}, t\right)}{\partial n}=u_{n}\left(\mathbf{x}_{\Gamma}, t\right)=0,
$$

with $\mathbf{x}_{\Gamma}$ is located at the tailpipe boundary, ground surface and automotive body, and $n$ is the direction normal to the boundary. For the axisymmetric problem of Fig. B.1, the axisymmetric form of the LEE and related source function and boundary conditions are solved. These are all given in Appendix A.

\subsection{Multi-domain extended Fourier pseudospectral time-domain method (PSTD)}

Equations (2) are solved by the PSTD method on an orthogonal equidistant grid, with velocity nodes staggered with the pressure and density nodes. The equations are marched in time by a low-storage optimized 6-stage Runge-Kutta method [23]. The spatial derivatives are computed using the extended Fourier pseudospectral method, which relies on an eigenfunction expansion of the LEE for a medium at rest and assuming isentropy [14]. The eigenfunctions are complex exponentials. Since fast 
Fourier transforms (FFTs) are used to calculate derivatives, only two spatial points per wavelength are needed with this method. Non-reflective boundaries are treated by including an absorption layer at the edges of the computational domain by means of the perfectly matched layer (PML); see [14]. For the axisymmetric case, the Fourier PSTD method does also apply to the $z$-direction. In the $r$-direction however, Bessel functions are more appropriate than the used complex exponential functions. As noticed by Gottlieb and Orszag [24], Bessel series converge at a slow rate, making them less attractive for spectral methods. As an alternative, a basis of Chebyshev or one-sided Legendre polynomials may be used, see [25]. The latter of these methods cannot benefit from using FFTs and the former is known to require more points per wavelength than the Fourier method and to require smaller time step by the clustering of points near the boundaries. To apply the Chebyshev method to the problem of Fig. B.1, the domain needs to be decomposed in sub-domains with a minimal dimension of $a$ for $r<a$. As the method typically needs 16 points per subdomain for convergence, this implies a large number of points and, consequently, a small time step for numerical stability. For calculations of the axisymmetric configuration, the Fourier pseudospectral method has therefore been used as well, both for the $z$ and $r$-direction. For the numerical solution of the LEE, the required grid spacing near the tailpipe exit is determined by the dimensions of the local geometry and mean flow gradients. This leads to a grid which is finer than the grid which is required for resolving the smallest acoustical wavelength of interest in the bulk of the domain. As a result, an orthogonal equidistant PSTD mesh over resolves most of the domain. Thus, from a computational point of view, it is attractive to apply a multi-domain approach, i.e. a fine grid in the vicinity of the tailpipe and a coarser grid elsewhere. For this reason, the multi-domain PSTD methodology, that has recently been published [19], is utilized for most of the calculations.

For the geometry of Fig. B.2(a), the total domain is composed of the coarse and fine grids without their overlapping areas, depicted by the large and small box in Fig. B.2(b). Coarse grid pressure and densities are staggered with their fine grid coun- 
terparts in all directions. The coarse sub-domain $\Omega_{c}$ covers the full domain, thereby extending outside the coarse-fine grid interface into the fine grid area (Fig. B.2(c,d)). In the overlapping region, data transfer takes place from the fine to the coarse grid. The fine sub-domain $\Omega_{f}$ extends outside the coarse-fine grid interface into the coarse grid. In the extended region, data transfer takes place from the coarse to the fine grid. In the outermost region of the fine grid, i.e. outside the region where data transfer takes place, values are multiplied by a super-Gaussian window function to obtain periodicity in $\Omega_{f}$. The coarse grid $\Omega_{c}$ is discretized with equidistant spacing $\Delta x_{c}$, the fine grid $\Omega_{f}$ with equidistant spacing $\Delta x_{f}=\Delta x_{c} / d f$ with $d f$ the integer discretization factor. The discrete time step of the coarse grid is $\Delta t_{c}=\frac{\Delta x_{c}}{2 c_{0}}$ [14], and the fine grid has time step $\Delta t_{f}=\frac{\Delta t_{c}}{d f}$. During one time iteration $\Delta t_{c}$, the fine grid results are iterated $d f$ times without data transfer with the coarse grid. After each $\Delta t_{c}$, data transfer takes place from coarse to fine grid and vice versa (Fig. B.2(c)). Coarse grid data are spectrally interpolated to the fine grid positions and fine grid data are decimated by $d f$ and spectrally shifted to the coarse grid positions. As the area modelled by the fine grid can lead to wavelengths which cannot be resolved by the coarse grid, a low pass filter is applied to the fine grid variables prior to decimation.

This multi-domain approach is shown not to introduce significant errors over a single PSTD method when an appropriate choice of the dimensions of the data transfer zones is made [19]. A super-Gaussian window, corresponding to an error of $-40 \mathrm{~dB}$, is chosen, along with a width of $0.2 \mathrm{~m}$ outside the sub-domain interfaces for the coarse to fine grid data transfer region (Fig. B.2(c)). The current multi-domain application is found to lead to large errors arising from a discrepancy of the aliasing error from both grids due to the wave appearing at the tailpipe origin, which acts as a point-like source. To successfully overcome this problem, the data transfer takes place with a linear interpolation, as illustrated in Fig. B.2(c). In this way, continuity of the variable values is promoted. 


\subsection{Reynolds-Averaged Navier-Stokes equations (RANS)}

The mean flow variables of Eqs. (2) are obtained by solving the RANS equations using commercial software. As shown in Appendix B, this approach is suitable for jet flows. Since the computational grid as used for the RANS solution differs from the orthogonal PSTD grid, a mapping of the RANS results needs to be applied. For this reason, the mean flow variables $\mathbf{q}_{0, R A N S}$ are mapped to the PSTD grid by applying a rectangular filter:

$$
\mathbf{q}_{0}(\mathbf{x})=\left[\begin{array}{ccc}
\frac{\sum_{i} \mathbf{q}_{0, R A N S}\left(\mathbf{x}_{i}\right) V\left(\mathbf{x}_{i}\right)}{\sum_{i} V\left(\mathbf{x}_{i}\right)} & \text { for } & \left|\mathbf{x}_{i}-\mathbf{x}\right| \leq 2 a / m \\
0 & \text { for } & \left|\mathbf{x}_{i}-\mathbf{x}\right|>2 a / m
\end{array}\right.
$$

with $i$ the index running over all nodes of the RANS solution mesh, $V_{i}$ the cell volume belonging to node $i$ and $m$ determining the spectral content of the mapping operation. A low value of $m$ smoothes the background flow components, which promotes stability of the numerical solution of the LEE, but can also suppress some relevant physical processes. On the other hand, a too high value of $m$ can lead to spurious oscillations in the numerical solution of the LEE as small wave numbers, which cannot be resolved by the discretization of the used PSTD methodology, are present in the solution. The effect of the choice of $m$ is shown in Section 4.2.2.

\subsection{Kirchhoff-Helmholtz integral equation (KHIE)}

To study the influence of the surrounding geometry and non-uniform mean flow and temperature on the directivity, far field results are needed. When assuming a nonmoving medium, a constant temperature and a rigid ground surface outside the outer volume of the computational domain as depicted in Fig. B.2(b), the KHIE is used to compute the solution at an arbitrary receiver position outside the PSTD domain ${ }^{1}$ :

$$
p_{i j}\left(\mathbf{x}_{r}\right)=\int_{S_{c}}\left(G_{i j}\left(\mathbf{x}_{r} \mid \mathbf{x}_{s r}\right) \frac{\partial p_{i j}\left(\mathbf{x}_{s r}\right)}{\partial n}-p_{i j}\left(\mathbf{x}_{s r}\right) \frac{\partial G_{i j}\left(\mathbf{x}_{r} \mid \mathbf{x}_{s r}\right)}{\partial n}\right) \mathrm{d} \mathbf{x}_{s r}
$$

\footnotetext{
${ }^{1} \mathrm{~A}$ small error is introduced by these assumptions as the maximum value of the flow velocity at the surfaces $S_{c}$ is $\mathrm{M}=0.02$ for the case of an inflow velocity of $\mathrm{M}=0.3$.
} 


$$
\begin{aligned}
G_{i j}\left(\mathbf{x}_{r} \mid \mathbf{x}_{s r}\right) & =\frac{e^{j k_{i j} R}}{4 \pi R}+\frac{e^{j k_{i j} R^{\prime}}}{4 \pi R^{\prime}}, \\
R & =\left|\mathbf{x}_{r}-\mathbf{x}_{s r}\right|, \\
R^{\prime} & =\left|\mathbf{x}_{r}-\mathbf{x}_{s r}^{\prime}\right|, \\
\mathbf{x}_{s r} & =\left[x_{s r}, y_{s r}, z_{s r}\right], \\
\mathbf{x}_{s r}^{\prime} & =\left[x_{s r}, y_{s r},-z_{s r}-0.6\right],
\end{aligned}
$$

with $p_{i j}\left(\mathbf{x}_{s r}\right)$ and $\frac{\partial p_{i j}\left(\mathbf{x}_{s r}\right)}{\partial n}$ obtained after Fourier transforming pressure and normal velocity results from the PSTD method with positions at the integration surfaces $S_{c}$, located at the outer volume of the computational domain as depicted in Fig. B.2(b). $R$ and $R^{\prime}$ are respectively the distance from the receiver position to a position at $S_{c}$ and to the image of the position at $S_{c}$ with the ground surface. Since Eq. (6) is evaluated by numerical quadrature, a higher spatial resolution as in the PSTD grid is needed to accurately compute the highest 1/3-octave band of interest. A grid spacing of $0.008 \mathrm{~m}$ is used, corresponding to a resolution of 17 points per wavelength for 2500 $\mathrm{Hz}$ in the absence of flow and for ambient temperature. This is found to lead to a maximum error of $0.4 \mathrm{~dB}$ for an analytical case. This resolution is obtained by spectrally interpolating the PSTD data at the surfaces $S_{c}$ prior to application of the Fourier transforms.

\section{Validity of PSTD approach}

To investigate the accuracy of the PSTD method for the current application, the axisymmetric tailpipe configuration in free field with and without the jet of Fig. B.1 is considered. The results of the calculations are compared with a numerical evaluation of an analytical expression as published by Levine and Schwinger [4], the Munt model as obtained by the algorithm from Gabard and Astley [26], as well as with results from the Discontinuous Galerkin (DG) method [27]. The calculations are initiated by the source function from Eqs. (A.2) with $z_{s}=-1 \mathrm{~m}, z_{s}=0 \mathrm{~m}$ being the end of the tailpipe. The computed time responses have been windowed to avoid aliasing 
effects prior to applying a discrete Fourier transform for obtaining the result in the frequency domain. The results are calculated at a distance $\mathbf{r}$ from the tailpipe exit and are presented as the sound pressure level relative to the acoustic pressure incident to the pipe exit (which has been multiplied by $1+M$ to relate it to the actual acoustic intensity), and normalized to a distance of $1 \mathrm{~m}$ behind the tailpipe by multiplying the ratio $\frac{P_{\mathrm{rad}}(\theta, f)}{P_{\mathrm{tp}}(f)(1+M)}$ by $\mathbf{r}$ :

$$
\begin{aligned}
L_{p}(\theta, f) & =20 \log _{10}\left|\frac{P_{\mathrm{rad}}(\theta, f) \mathbf{r}}{P_{\mathrm{tp}}(f)(1+M)}\right|, & \\
P_{\mathrm{rad}}(\theta, f) & =\mathcal{F}(p(\mathbf{r} \sin (\theta), \mathbf{r} \cos (\theta), \mathbf{t})), & \\
P_{\mathrm{tp}}(f) & =\mathcal{F}\left(p\left(r_{\mathrm{tp}}, z_{\mathrm{tp}}, t\right)\right), & 0<t<3 \mathrm{~ms}
\end{aligned}
$$

with $r_{\mathrm{tp}}=0 \mathrm{~m}$ and $z_{\mathrm{tp}}=-0.4 \mathrm{~m}$ the tailpipe position, $\mathcal{F}$ the forward Fourier transform

and $\theta$ the angle according to Fig. B.1. For all computations $\Delta t_{\mathrm{PSTD}}=\frac{\Delta z_{\mathrm{j}, \mathrm{PSTD}}}{2 c_{0}}$ has been used. The signal in the tailpipe has been recorded for $0<t<3 \mathrm{~ms}$, such that only the rightward travelling wave incident to the tailpipe edge is captured: the wave travelling leftward in the pipe is absorbed by the PML and the wave reflected from the tailpipe edge arrives later than $3 \mathrm{~ms}$.

\subsection{Tailpipe without jet}

Figure B.3 shows results for the (octave band center) frequencies $63 \mathrm{~Hz}$ and 2000 $\mathrm{Hz}$, from PSTD calculations for the three equidistant discretizations, i.e. $\Delta z=2 a$, $\Delta z=a$ and $\Delta z=a / 2$, analytical results [4] and DG results [27]. The angular dependency for the PSTD results is similar to the analytical and DG results, yet the levels deviate. Results are within $0.3 \mathrm{~dB}$ from the analytical results for $\Delta z=a / 2$. The high discretization needed in the PSTD method can be attributed to the used complex exponential functions in $r$-direction instead of Bessel functions. This is confirmed by results of calculations for the configuration of the $3-\mathrm{D}$ rectangular exhaust pipe in free field, see Fig. B.10. The DG results are shown in Fig. B.4, along with PSTD results for three values for $\Delta z$. The PSTD results clearly converge more rapidly with $\Delta z$ than for the axisymmetric case. 


\subsection{Tailpipe with jet}

\subsubsection{Numerical stability}

Figure B.5 shows snapshots of the solution at two time instances from PSTD calculations. In Fig. B.5(a), the wave incident from the exhaust pipe has propagated about $1.5 \mathrm{~m}$ from the pipe exit. Its oscillating nature is due to dispersion. The wavelet close to the axis corresponds to the hydrodynamic wave which is excited at the tailpipe edge. To verify this assumption, the vorticity level $L_{\omega}$ is shown in Figs. B.5(c,d), indicating that the pressure wave is associated with shed vorticity. In Fig. B.5(b), it can be noticed that the acoustic wave has propagated outside the shown domain. An additional pressure field with the pipe exit as source position appears and eventually leads to instability. The start of this instability can be found by monitoring the acoustic energy in the high wavenumber components. In the applied Fourier PSTD method, the acoustic energy should decrease towards the highest resolved wavenumber to avoid aliasing. Therefore, when the energy in the high wavenumber region exceeds the energy in a region of lower wavenumbers, the risk for instability is large. In the current configuration, the wavenumber spectrum of the pressure at $r=a-\Delta x / 2$ is computed at every time step. Then, a filter has been applied after a time step $t$ for which the following condition holds

$$
\sum_{n=(1-\delta) \frac{k_{\max }}{\Delta k}}^{n=\frac{k_{\max }}{\Delta k}}\left|p_{r}^{t}(n \Delta k)\right|^{2}>\sum_{n=(1-2 \delta) \frac{k_{\max }}{\Delta k}}^{n=(1-\delta) \frac{k_{\max }}{\Delta k}}\left|p_{r}^{t}(n \Delta k)\right|^{2}
$$

with $k_{\max }=\pi / \Delta z, p_{r}^{t}(n \Delta k)$ the wave number spectrum of the pressure at $r=a-\Delta x / 2$ and time $t$, and with $\delta$ a small number. A value of $\delta=0.05$ has been used here which gives stable and accurate results. The acoustic variables are filtered according to the 
following low-pass filter:

$$
Q_{n_{r}, n_{z}}=\left[\begin{array}{lll}
Q_{n_{r}, n_{z}} \mathrm{e}^{-\frac{25}{2}\left(\frac{\left|n_{r}\right| \Delta k-k_{c}}{k_{\max }-k_{c}}\right)^{2}-\frac{25}{2}\left(\frac{\left|n_{z}\right| \Delta k-k_{c}}{k_{\max }-k_{c}}\right)^{2}} & \text { for } \quad k_{c} / \Delta k<\left|n_{r}\right|,\left|n_{z}\right| \leq k_{\max } / \Delta k \\
Q_{n_{r}, n_{z}} \mathrm{e}^{-\frac{25}{2}\left(\frac{\left|n_{r}\right| \Delta k-k_{c}}{k_{\max }-k_{c}}\right)^{2}} & \text { for } \quad k_{c} / \Delta k<\left|n_{r}\right| \leq k_{\max } / \Delta k \\
Q_{n_{r}, n_{z}} \mathrm{e}^{-\frac{25}{2}\left(\frac{\left|n_{z}\right| \Delta k-k_{c}}{k_{\max }-k_{c}}\right)^{2}} & \left|n_{z}\right| \leq k_{c} / \Delta k \\
& \text { for } \quad k_{c} / \Delta k<\left|n_{z}\right| \leq k_{\max } / \Delta k \\
Q_{n_{r}, n_{z}} & \left|n_{r}\right| \leq k_{c} / \Delta k \\
& \text { for } \quad\left|n_{r}\right|,\left|n_{z}\right| \leq k_{c} / \Delta k
\end{array}\right.
$$

with $k_{c}$ the cut-off wave number. As derivatives in the PSTD method are computed in the wave number domain, it is a straightforward procedure to apply this filtering to the wave number domain variables $Q$. The filter has zero dissipation up to $k_{c}$ and a Gaussian shaped decay beyond that. As a result, the acoustic variables up to the frequency of interest are not dissipated and only small wave number components are attenuated. Figure B.6 show the results with the applied filtering. Clearly, the instability is removed.

\subsubsection{Grid convergence and mean flow filtering aspects}

From the computed impulse responses at $2 \mathrm{~m}$ from the pipe exit, $L_{p}$ values are again computed according to Eq. (7). In the Figures B.7, B.8 and B.9, PSTD results are compared with results from the Munt model, as obtained by the algorithm from Gabard and Astley [26]. From this analytical model, the results with the full Kutta condition (all possible vorticity shed) and the no Kutta condition (no vorticity shed) are shown. Figs. B.7(a,b) illustrate the influence of the PSTD discretization, with $\Delta z=a / j$ and $j=2,4$ and 8 . Further, $m=8$ and $k_{c}=\pi / a$ have been used. The results converge for the finer discretization and are close to the analytical results with

the full Kutta condition, which justifies the adopted LEE approach and the applied PSTD method. For the $2 \mathrm{kHz}$ results, the deviations for the lowest angles are caused by the fact that the Munt model assumes the jet flow to be uniform for $0 \leq r \leq a$ and for all $z$. The better agreement for $\Delta x=a / 2$ than for $\Delta z=a / 8$ at the highest angles for $2000 \mathrm{~Hz}$ are caused by the fact that the used filter for stability $k_{c}$ is too coarse, as 
can be seen in Fig. B.8. Whereas $\Delta z=a / 2$ was shown to be sufficient to solve the tailpipe problem for a quiescent medium, a discretization of $\Delta z=a / 8$ is needed for accurate results in the presence of the jet flow due to the high gradients in the mean flow. As the grid is too fine for most of the domain, numerical efficiency of the PSTD is sub-optimal. Therefore, the multi-domain PSTD methodology is applied, with the fine grid $\Delta z=a / 8$ used in the region with the high mean flow gradients, a coarser grid $\Delta z=a / 2$ used outside this area. All further computations with PSTD in this paper are carried out using the multi-domain methodology as presented in Section 3.2. The effect of the filter on the acoustic variables as imposed to obtain numerical stability is shown in Fig. B.8 for $k_{c}=\pi /(2 a), \pi / a$ and $2 \pi / a$, and with $m=8, \Delta z_{c}=a / 2$ and $\Delta z_{f}=a / 8$. A lower $k_{c}$ number clearly reduces the accuracy of the results as a part of the physical processes are discarded when using the filter. The choice of $k_{c}=2 \pi / a$ is used further. The effect of the mean flow filter is demonstrated in Fig. B.9 for $m=2,4$ and 8 , and with $\Delta z_{c}=a / 2, \Delta z_{f}=a / 8$ and $k_{c}=2 \pi / a$. A lower value of $m$ is smoothing the flow effects and the results show a lower directionality.

\section{Results}

Results are computed for three configurations, depicted in Fig. B.10, and for three flow conditions: no jet, a cold jet and a hot jet. The source position is located at $x=-1.52 \mathrm{~m}$ in Fig. B.2(b). The 3-D LEE are solved for this configuration, with a filter applied to the acoustic variables with cut-off wavenumber $k_{c}=2 \pi / a, \Delta x_{f}=a / 2$, $\Delta x_{c}=2 a$ and $m=8$. The choices of $\Delta x_{f}=a / 2, \Delta x_{c}=2 a$ rely on the 3D results in Section 4.1. Of interest are the radiated power and directivity. The radiated power level results are computed as:

$$
\begin{aligned}
L_{w, j} & =10 \log _{10}\left(\frac{\sum_{i=1}^{10} W_{r a d, i j}}{\sum_{i=1}^{10} W_{i n, i j}}\right), \\
W_{r a d, i j} & =\frac{1}{2} \int \mathcal{R}\left[p_{i j} \overline{v_{i j}}\right] \cdot \overrightarrow{\mathbf{n}} \mathrm{d} S_{r}, \\
W_{i n, i j} & =\frac{S_{p}(1+M)^{2}}{2 \rho_{0} c_{0}}\left|p_{i n, i j}\right|^{2},
\end{aligned}
$$


with $W_{\text {rad }}$ the radiated power and $W_{i n}$ the power incident in the pipe, $p_{i n, i j}$ the pressure component of the incident sound wave and $M, \rho_{0}$ and $c_{0}$, respectively, the Mach number, density and speed of sound in the pipe. The cross sectional area of the pipe is denoted by $S_{p}$. The overbar denotes the complex conjugate and the integral is over $S_{r}$, the receiver planes surrounding the geometry illustrated by the outer volume of Fig. B.2(b). The indices $j$ and $i$ represent the $1 / 3$-octave band number and the frequencies within the $1 / 3$-octave bands. For the latter, 10 frequencies have been chosen. Isentropic conditions in the pipe are assumed for the computation of $W_{i n}$.

Directivity results are defined as the sound pressure level relative to the maximum sound pressure level for a certain 1/3-octave band:

$$
L_{p, j}(R, \phi, \theta)=10 \log _{10}\left(\frac{\sum_{i=1}^{10}\left|p_{i j}(R, \phi, \theta)\right|^{2}}{\max _{\phi, \theta}\left(\sum_{i=1}^{10}\left|p_{i j}(R, \phi, \theta)\right|^{2}\right)}\right),
$$

where $R$ is the fixed distance of the receiver plane, $\phi$ the horizontal angle and $\theta$ the vertical angle, see e.g. Fig. B.10.

\subsection{Tailpipe without jet}

In the no jet case, $L_{w, j}$ is computed and presented in Fig. B.11. For the configuration in free field, the radiated power linearly increases with frequency (Fig. B.11(a)). Analytical results [28] for the radiated power of an unflanged circular pipe with iden-

tical cross section as the rectangular pipe of the current study, i.e. a radius of $\sqrt{a^{2} / \pi}$, are plotted in the same subfigure. The results are in good agreement. Slight deviations at high frequencies can be caused by the fact that the pipe under consideration is not circular.

For the configuration with the ground surface, the radiated power is higher than for the free field case for low frequencies (Fig. B.11(b)). The low frequency results reach the solution of a pipe with twice the cross section, as predicted by an analytical calculation. As the ratio of the height of the tailpipe over the ground surface over the wavelength increases with frequency, the radiated power reduces to the radiated power for the free field case for frequencies above $250 \mathrm{~Hz}$. The presence of the body 
further increases the radiated power (Fig. B.11(c)). The analytical result, plotted by the dashed line, represents a flanged circular pipe orifice with twice the physical cross section. The surrounding geometry thus causes an increase of about $6 \mathrm{~dB}$ in the lower frequency region in comparison to the free field case.

Directivity results are plotted per configuration in Figs. B.12, B.13 and B.14. The upper halves of the polar plots display the directivity in the $x, z$-plane at $y=0 \mathrm{~m}$, and the lower halves the directivity in the $x, y$-plane at $z=0 \mathrm{~m}$. Due to symmetry reasons, only half of the polar plots are shown for the free field case in Figs. B.12. The receiver positions are located at a distance of $50 \mathrm{~m}$ from the orifice of the pipe. Two 1/3-octave bands are selected for the directivity plots. For the free field case in Fig. B.12, analytical results for an unflanged pipe without jet are plotted along with the PSTD results. For the case with the ground and ground and body in respectively Figs. B.13 and B.14, analytical results of a point source over a ground surface are shown along with the PSTD results. The results without jet are discussed first. For the case without jet in free field, the $63 \mathrm{~Hz}$ 1/3-octave band shown in Fig. B.12(a) shows an almost omnidirectional pattern. The ground surface does not significantly change the directivity in contrast to the automotive body, see Figs. B.13(a) and B.14(a). A shielding effect is noticed in front of the body and an interference effect occurs above it.

The $2000 \mathrm{~Hz}$ band is slightly more directional for the free field case with highest values behind the pipe exit, see Fig. B.12(b). Differences with analytical results as computed according to reference [4] can have the same nature as those discussed from the sound power results. In the presence of the ground surface as shown in Fig. B.13(b), interference effects are clearly visible. The computed interference pattern matches the analytical directivity of a point source over a ground surface. Fig. B.14(b) shows the influence of the automotive body, which strongly influences the directivity with high shielding in front of the body. 


\subsection{Tailpipe with jet}

Directivity plots for the cases with cold and hot flow are included for the three configurations, and are shown in, respectively, subfigures (c), (d), (e) and (f) of Figs. B.12, B.13 and B.14. For the configuration in free field and with ground only, the $63 \mathrm{~Hz}$ band has the highest levels in the region behind the pipe and decreases with increasing angle with a reduction up to about $10 \mathrm{~dB}$. The effect is similar for the cold and hot jets. The directivity pattern in the presence of ground, body and flow, shown in Figs. B.14(c) and (e), mimics the results from the same configuration without flow. The additional flow effect is moderate but clearly visible, also in the area geometrically screened from the tailpipe orifice.

For the $2000 \mathrm{~Hz}$ band, a cone of low sound levels is created behind the pipe, and is visible for both cold and hot jets in all three configurations. This effect is more pronounced for the hot flow than for the cold flow. For the ground case, it can be noticed that the ground interference pattern is still present, but the amplitudes are modified by the flow effects. For the ground-body configuration, the main additional effect of the flow on the directivity is in the region behind the exhaust pipe. The geometrically shielded area is less influenced by the flow.

Figs. B.15 show the directivity for the ground-body case for all receiver positions in a hemisphere at $R=50 \mathrm{~m}$ around the configuration by a top view. These results emphasize that both flow and surroundings determine the directivity. The result for the $2000 \mathrm{~Hz}$ 1/3-octave band in Figs. B.15(b), (d) and (f) clearly show the ground interferences, an interference with the reflected sound from the body and a the shielding effect. Finally, radiated noise is studied for the configuration with ground and body for a range of inflow velocities and inlet temperatures representing various automotive exhaust engine speeds according to Fig. 17 from [21]. Five non-zero inlet velocities have been chosen $(32,54,75,92$ and $108 \mathrm{~m} / \mathrm{s})$ with an ambient temperature and the same velocities have been chosen with increasing inlet temperature $(250,300,350$, 400 and $450 \mathrm{~K}$ ). The radiated sound pressure level in the $2000 \mathrm{~Hz} 1 / 3$ octave band is computed for these cases, as Figs. B.12, B.13, B.14 and B.15 show that flow effects 
are large for this $1 / 3$ octave band. The radiated level is computed as

$$
L_{p, r a d}(R, \phi, \theta)=10 \log _{10}\left(\frac{\sum_{i=1}^{10}\left|p_{i j}(R, \phi, \theta)\right|^{2}}{\frac{\left(\rho_{0} c_{0}\right)_{r e f}(1+M)^{2}}{\rho_{0} c_{0}} \sum_{i=1}^{10}\left|p_{i n, i j}\right|^{2}}\right),
$$

with $j$ the index for the $2000 \mathrm{~Hz}$ octave band. $L_{p \text {,rad }}$ is relative to the pressure level incident in the pipe that is normalised to its acoustic power. Fig. B.16 shows $L_{p, \text { rad }}$ for $R=50 \mathrm{~m}$ and $\theta=0 \mathrm{deg}$. The results for $\theta=0 \mathrm{deg}$. have been chosen as

radiation in the horizontal plane is most relevant for environmental noise analysis of an automotive exhaust. Fig. B.16(a) and (b) show $L_{p, r a d}$ for $\phi=-5,-90$ and -175 deg., and Fig. B.16(c) and (d) show $L_{p, r a d}$ for the whole range of angles $\phi$. For the cold jet, Fig. B.16(a) clearly displays that the level behind the exhaust pipe at $\phi=-5$ deg. gradually decreases for higher inlet velocities. The levels at $\phi=-175$ does show a weaker decrease of the level with increasing flow velocity whereas the levels at $\phi=$ -90 do not show a much dependency on the inflow velocity. The results for the hot jet show that the higher temperature causes significantly lower noise levels already from inlet flow velocities of $32 \mathrm{~m} / \mathrm{s}$. Fig. B.16(c) also shows that the combined effect of temperature and jet flow causes a pronounced area of low noise levels behind the exhaust pipe, whereas the influence of the higher temperature is not high for the other angles.

\section{Conclusions}

The present work numerically investigates the effects of exhaust jets with nonuniform mean flow and temperature as well as geometrical effects on the radiated sound field from automotive exhaust pipes. This study focusses on a 3-D configuration with a rigid ground surface and an automotive body, represented by a rigid cuboid. A 2-step computational approach is adopted, where the mean jet flow is computed by a RANS simulation and the sound field (with sources inside the exhaust pipe) is propagated through the mean flow field by solving the LEE equations. The applicability of solving the LEE by an highly efficient method, the Fourier pseudospectral time-domain (PSTD) method, is studied first. The PSTD method shows to give 
accurate results when compared to reference results for the case without jet, although a higher number of degrees of freedom are required for the axisymmetric problem than for a Cartesian problem due to the approximate functions to solve the spatial derivatives in the method for the studied axisymmetric case. Translating the mean flow field results to the acoustic grid corresponds to applying a filtering operation. It is concluded that a too smoothly resolved flow field leads to an underestimation of the effect of the jet flow. Numerical instabilities appear when solving LEE by PSTD in the presence of an exhaust jet. The onset of this instability can be detected by monitoring the acoustic energy at the high wave numbers and are suppressed by filtering out these high wave number components, although the accuracy of the results is sensitive to the chosen filter. In the presence of the jet, a higher discretization is needed in PSTD to resolve the occurring physical phenomena than for the no-jet case, and the multi-domain PSTD method has been used successfully by locally refining the grid. Results of the LEE solved by PSTD in the presence of the jet show a good agreement with reference results. For the 3-D configuration with the ground and body, it is shown that, for the low frequency region where noise from the combustion engine has its main contributions, the geometrical effects lead to an increase of the radiated sound power by $6 \mathrm{~dB}$ compared to an exhaust pipe in free field. The body causes a shielding of the radiated noise, which is more pronounced at higher frequencies. In the absence of the rigid body, the presence of an exhaust flow causes a clear directivity effect, with typically $-10 \mathrm{~dB}$ lower levels in the upstream direction, both for the low and high frequencies. This effect is still visible in the presence of the body. At high frequencies, a cone of low noise levels is created behind the exhaust pipe due to the presence of a jet flow, with similar effects for the configurations with and without body. The influence of the jet has been studied for a range of velocity and temperature values and the convective directivity effects are concluded to be more pronounced for a hot flow compared to a cold flow. 


\section{Acknowledgements}

The authors are grateful to Dr. Gabard for providing results from the Munt model and to the anonymous reviewers of this manuscript for their useful comments. This research was supported by a Marie Curie Intra European Fellowship within the 7th European Community Framework Programme.

\section{Appendix A. Axisymmetric linearized Euler equations}

For the axisymmetric problem of Fig. B.1, the axisymmetric linearized Euler equations are solved. The $(r, \theta, z)$ coordinate system with fluctuation velocities $\left(u_{r}, u_{\theta}, u_{z}\right)$ and mean flow velocities $\left(u_{0, r}, u_{0, \theta}, u_{0, z}\right)$ is adopted and axisymmetry is assumed, i.e. $u_{\theta}=u_{0, \theta}=0$. The non-conservative form can under isentropic conditions be formulated as:

$$
\begin{aligned}
& \frac{\partial \mathbf{q}_{\mathrm{axi}}}{\partial t}+\mathbf{A}_{\mathbf{j}} \frac{\partial \mathbf{q}_{\mathrm{axi}}}{\partial j}+\mathbf{C q}_{\mathrm{axi}}+\frac{\mathbf{H q}_{\mathrm{axi}}}{r}=\mathbf{q}_{\mathrm{s}, \mathrm{axi}}, \\
& \mathbf{A}_{j}=\left[\begin{array}{cccc}
u_{0, j} & \rho_{0} \delta_{r, j} & \rho_{0} \delta_{z, j} & 0 \\
0 & u_{0, j} & 0 & \frac{\delta_{r, j}}{\rho_{0}} \\
0 & 0 & u_{0, j} & \frac{\delta_{z, j}}{\rho_{0}} \\
\mathbf{C}= & {\left[\begin{array}{cccc}
0 & \gamma p_{0} \delta_{r, j} & \gamma p_{0} \delta_{z, j} & u_{0, j}
\end{array}\right],} \\
\frac{u_{0, j}}{\rho_{0}} \frac{\partial u_{0, r}}{\partial j} & \frac{\partial u_{0, r}}{\partial r} & \frac{\partial u_{0, r}}{\partial z} & 0 \\
\frac{u_{0, j}}{\rho_{0}} \frac{\partial u_{0, z}}{\partial j} & \frac{\partial u_{0, z}}{\partial r} & \frac{\partial u_{0, z}}{\partial z} & 0 \\
0 & \frac{\partial p_{0}}{\partial r} & \frac{\partial p_{0}}{\partial z} & 0
\end{array}\right] \\
& \mathbf{H}=\left[\begin{array}{cccc}
0 & 0 & 0 \\
0 & \rho_{0} & 0 & 0 \\
0 & 0 & 0 & 0 \\
0 & 0 & 0 & 0 \\
0 & \gamma p_{0} & 0 & 0
\end{array}\right],
\end{aligned}
$$

with $\mathbf{q}_{\text {axi }}=\left[\rho, u_{r}, u_{z}, p\right]^{T}$, index $j$ equals $r$ or $z, \mathbf{q}_{\mathrm{s}, \mathrm{axi}}$ is the source vector, $\gamma$ the heat capacity ratio and $\delta_{i, j}$ the Kronecker delta. Equations (A.1) are accompanied by the 
following source function and boundary conditions:

$$
\begin{gathered}
\mathbf{q}_{\mathrm{s}, \text { axi }}(\mathbf{r}, t)=\left[\begin{array}{c}
\frac{\delta\left(z-z_{s}\right)}{c_{0}^{2}} \sin \left(2 \pi f_{s} t\right) e^{-b\left(t-t_{s}\right)^{2}} \\
0 \\
0
\end{array}\right] \text { for } \quad r<a, \\
\left.\mathbf{q}_{\mathrm{s}, \text { axi }}(\mathbf{r}, t)=0 \begin{array}{c}
\text { for } \quad r>a, \\
\delta\left(z-z_{s}\right) \sin \left(2 \pi f_{s} t\right) e^{-b\left(t-t_{s}\right)^{2}}
\end{array}\right] \\
\frac{\partial p\left(\mathbf{r}_{\Gamma}, t\right)}{\partial n}=u_{r}\left(\mathbf{r}_{\Gamma}, t\right)=0,
\end{gathered}
$$

with $\mathbf{r}=(r, z)$. For $r=0$, l'Hôpital's rule has been used to avoid the singular terms:

$$
\begin{aligned}
& \frac{\partial \mathbf{q}_{\text {axi }}}{\partial t}+\mathbf{A}_{\mathbf{j}} \frac{\partial \mathbf{q}_{\mathrm{axi}}}{\partial j}+\mathbf{C q}_{\mathrm{axi}}=\mathbf{q}_{\mathrm{s}, \mathrm{axi}}, \\
& \mathbf{A}_{j}=\left[\begin{array}{cccc}
u_{0, j} & 2 \rho_{0} \delta_{r, j} & \rho_{0} \delta_{z, j} & 0 \\
0 & u_{0, j} & 0 & \frac{\delta_{r, j}}{\rho_{0}} \\
0 & 0 & u_{0, j} & \frac{\delta_{z, j}}{\rho_{0}} \\
0 & 2 \gamma p_{0} \delta_{r, j} & \gamma p_{0} \delta_{z, j} & u_{0, j}
\end{array}\right], \\
& \mathbf{C}=\left[\begin{array}{cccc}
\frac{u_{0, j}}{\rho_{0}} \frac{\partial u_{0, r}}{\partial j} & \frac{\partial u_{0, r}}{\partial r} & \frac{\partial u_{0, r}}{\partial z} & 0 \\
\frac{u_{0, j}}{\rho_{0}} \frac{\partial u_{0, z}}{\partial j} & \frac{\partial u_{0, z}}{\partial r} & \frac{\partial u_{0, z}}{\partial z} & 0 \\
0 & \frac{\partial p_{0}}{\partial r} & \frac{\partial p_{0}}{\partial z} & 0
\end{array}\right],
\end{aligned}
$$

\section{Appendix B. RANS simulation of a free jet}

A mean velocity field in the geometry of interest is generated by performing a RANS calculation, for which the program ANSYS/FLUENT has been used. FLUENT is used in the Workbench of the ANSYS 12 package, where the DesignModeler has been used to generate the geometry and the ANSYS Meshing is used to generate the mesh. To calibrate the model in FLUENT, benchmark measurements of a jet are utilized first. The benchmark data have been taken from the AGARD test cases, i.e. 
case SHL30 [29]. For the settings in FLUENT, use has been made of the FLUENT tutorial, best practice guidelines from the European research community on flow, turbulence and combustion [30], and from FLUENT settings as published for a similar geometry [31].

\section{Appendix B.1. Calibration of the model AGARD test case SHL30}

The SHL30 results consist of profiles of the axial velocity for distances in the selfsimilarity region of the flow, i.e. $z / D>30$ for the current configuration, and the decay of the axial velocity on the $z$-axis over the distance in the self-similarity region. For the RANS modelling, the settings of Table B.1 have been used. The standard $C_{2}$ coefficient in the $\kappa-\epsilon$ model amounts to 1.92. Following arguments from [31], a lower value is used to improve the results, and 1.87 has been used here. The standard $\kappa-\epsilon$ model is known to be erroneous for free jets. Following the FLUENT instructions, the realizable $\kappa-\epsilon$ model should better represent free jets. It is however found that the $\kappa-\epsilon$ model provides better results for the current application. The obtained results are found to be consistent with results of a finer mesh and a larger domain. Fig. B.17(a) shows the on-axis mean axial velocity from the AGARD measurements and from FLUENT as a function of the distance. The agreement is good. Figure B.17(b) shows the velocity profiles as a function of the radial distance, normalized to $r_{1 / 2}$, the half jet flow width, i.e. $r$ where $u_{0, z}=u_{0, z, c} / 2$, with $u_{0, z, c}$ the on-axis axial velocity. The results are plotted for the subsequent distances $0.8 \mathrm{~m}-2.8 \mathrm{~m}$ with an interval of $0.4 \mathrm{~m}$ and are found to be in good agreement with the measurements.

\section{Appendix B.2. Tailpipe geometry}

Most settings from the SHL test case, discussed above, are used to generate the mean velocity profiles of the tailpipe geometry of Fig. B.1. The differences with above settings are listed in Table B.2. Figure B.18 shows a 2-D plot of the mean axial velocity, where the rectangular filter of Eq. (5) has been used with $m=2$. The tailpipe has an elevation of $0.3 \mathrm{~m}$ in the application with the ground surface. As free 
field RANS results show very low flow velocities at $0.3 \mathrm{~m}$ below the pipe, the free field RANS results have been used for the case with the ground too.

[1] Den Boer, LC, Schroten, A. Traffic noise reduction in Europe: Health effects, social costs and technical and policy options to reduce road and rail traffic noise. Report: CE Delft, The Netherlands; 2007.

[2] Jonasson $\mathrm{H}$ et al.. Source modelling of road vehicles. Deliverable 9 of the HARMONOISE project, report HAR11TR-041210-SP10, Borås, Sweden; 2004.

[3] Peeters, B, van Blokland, G. The Noise Emission Model For European Road Traffic. Deliverable 11 of the IMAGINE project, report IMA55TR-060821-MP10; 2007.

[4] Levine, H, Schwinger, J. On the radiation of sound form an unflanged circular pipe. Phys Rev 1948;73(4):383-406.

[5] Munt, RM. The interaction of sound with a subsonic jet issuing from a semiinfinite cylindrical pipe. J Fluid Mech 1977;83(4):609-40.

[6] Munt, RM. Acoustic transmission properties of a jet pipe with subsonic jet flow, I: the cold jet reflection coeffcient. J Sound Vib 1990;142(3):413-36.

[7] Kok, JC. Computation of sound radiation from cylindrical ducts with jets using a high-order finite-volume method. In: Proceedings of the 13th AIAA/CEAS Aeroacoustics Conference, Rome, Italy; 2007, AIAA paper 2007-3489.

[8] Özyörük, T, Dizemeny, E, Kayay, E, Aktürkz, A. Frequency Domain Linearized Euler Solver for Turbomachinery Noise Propagation and Radiation. In: Proceedings of the 13th AIAA/CEAS Aeroacoustics Conference, Rome, Italy; 2007, AIAA paper 2007-3521.

[9] Zhang, X, Chen, X, Morfey, CL. Acoustic radiation from a semi-infinite duct with a subsonic jet. Int J of Aeroacoust 2005;4(1,2):169-84. 
[10] Agarwal, A, Morris, PJ, Mani, R. Calculation of sound propagation in nonuniform flows: suppression of instability waves. AIAA J 2004;42(1):80-88.

[11] Botteldooren, D. Finite-difference time-domain simulation of low-frequency room acoustic problems. J Acoust Soc Am 1995;98:3302-8.

[12] Manera, J, Schiltz, B, Leneveu, R, Caro, S, Jacqmot, J. Kelvin-Helmholtz instabilities occurring at a nacelle exhaust. In: Proceedings of the 14th AIAA/CEAS Aeroacoustics Conference, Vancouver, Canada; 2008, AIAA paper 2007-2883.

[13] Simonaho, SP, Lhivaara, T, Huttunen, T. Modeling of acoustic wave propagation in time-domain using the discontinuous Galerkin method - A comparison with measurements, Appl Acoust 2012;73:173-83.

[14] Hornikx, M, Waxler, R, Forssén, J. The extended Fourier pseudospectral time-domain method for atmospheric sound propagation. J Acoust Soc Am 2010;128(4):1632-46.

[15] Bashir, I, Hill, T, Taherzadeh, S, Attenborough, K, Hornikx, M. Reduction of Surface Transport Noise by Ground Roughness. Appl Acoust 2014;83:1-15.

[16] Hornikx, M, Forssén, J, Botteldooren, D, Van Renterghem, T, Wei, W, Ogren, M, Salomons, E. Urban background noise mapping: the multiple-reflection correction term. Acta Acust united Ac 2014;100(2):293-305.

[17] Van Renterghem, T, Hornikx, M, Forssen, J, Botteldooren, D. The potential of building envelope greening to achieve quietness. Build. Environ. 2012;61:34-44.

[18] Hornikx, M, Hak, C, Wenmaekers, R. Acoustic modelling of sports halls, two case studies. J. Build. Perform. Simu. 2015;8(1):26-38.

[19] Hornikx, M, De Roeck, W, Desmet, W. A multi-domain Fourier pseudospectral method for the linearized Euler equations, J Comp Phys 2012;231:4759-74. 
[20] Torregrosa, AJ, Broatch, A, Bermudez, V, Andres, I. Experimental assessment of emission models used for IC engine exhaust noise prediction. Exp Therm Fluid Sci 2005;30:97-107.

[21] Van der Walt, DC. Measurement technique to assess the acoustic properties of a silencer component for transient engine conditions. J Sound Vib 2001;243:797821.

[22] Davies, POAL, Holland, KR. The Measurement and Prediction of Sound Waves of Arbitrary Amplitude in Practical Flow Ducts. J Sound Vib 2004;271:849-61.

[23] Bogey, C, Bailly, C. A family of low dispersive and low dissipative explicit schemes for flow and noise computation. J Comp Phys 2004;194:194-214.

[24] Gottlieb, D, Orszag, SA. Numerical Analysis of Spectral Methods. Philadelphia, PA, SIAM; 1977.

[25] Boyd J. Chebyshev and Fourier Spectral Methods. Second edition, Dover, New York; 2001.

[26] Gabard, G, Astley, RJ. Theoretical model for sound radiation from annular jet pipes: far- and near-field solutions. J Fluid Mech 2006;549:315-41.

[27] Toulorge, T, Desmet, W. Curved Boundary Treatments for the Discontinuous Galerkin Method Applied to Aeroacoustic Propagation. AIAA J 2010;48(2):47989.

[28] Silva, F, Guillemain, P, Kergomard, J, Mallaroni, B, Norris, AN. Approximation formulae for the acoustic radiation impedance of a cylindrical pipe. J Sound Vib 2009;322:255-63.

[29] www.cfd-online.com/Links/refs.html

[30] Casey, M, Wintergerste W (eds.). Best Practice Guide. ERCOFTAC special interest group on "quality and trust in indutrial CFD". Version 1.0; 2000. 
[31] Azarpeyvand, M. Some aspects of RANS based jet noise prediction. Doctoral thesis, University of Southampton, Institute of Sound and Vibration, UK; 2008. 
Table B.1: FLUENT settings, AGARD case.

\begin{tabular}{|c|c|}
\hline Geometry & \\
\hline Domain size: $\mathrm{H} \times \mathrm{W}$ & $1.2 \mathrm{~m} \mathrm{x} 10 \mathrm{~m}$ \\
\hline Tailpipe radius & $12.7 \mathrm{~mm}$ \\
\hline Tailpipe wall thickness & $1 \mathrm{~mm}$ \\
\hline Tailpipe length & $500 \mathrm{~mm}$ \\
\hline Mesh Details & \\
\hline Minimum element size & $5 \mathrm{e}^{-5} \mathrm{~m}$ \\
\hline Maximum element face size & $5 \mathrm{e}^{-3} \mathrm{~m}$ \\
\hline Tailpipe walls edge sizing subdivisions & 40 \\
\hline Inflation applied to the walls of the tailpipe & \\
\hline with first layer height & $5 \mathrm{e}^{-5} \mathrm{~m}$ \\
\hline Maximum number of wall layers & 15 \\
\hline Number of mesh elements & 72,699 \\
\hline FLUENT settings & \\
\hline Axisymmetric mode & \\
\hline Standard $\kappa-\epsilon$ model & \\
\hline Standard wall functions & \\
\hline Air density & $1.2 \mathrm{~kg} / \mathrm{m}^{3}$ \\
\hline Axial inlet velocity in pipe & $56.2 \mathrm{~m} / \mathrm{s}$ \\
\hline Turbulent intensity & $0.58 \%$ \\
\hline Hydraulic diameter (turbulence property) & $25.4 \mathrm{~mm}$ \\
\hline Boundary conditions at other in- and outlets & \\
\hline$\kappa$ & $0.1 \mathrm{~m}^{2} / \mathrm{s}^{2}$ \\
\hline$\epsilon$ & $0.1 \mathrm{~m}^{2} / \mathrm{s}^{2}$ \\
\hline$C_{2}$ & 1.87 \\
\hline Roughness constant & 0 \\
\hline Slip wall conditions for the inner pipe surface & \\
\hline (to obtain a top-hatted profile as in the AGARD measurements) & \\
\hline Second order methods used for spatial and time derivatives & \\
\hline Initialization values: & \\
\hline Turbulent properties & from pipe inlet \\
\hline Axial velocity & $5 \mathrm{~m} / \mathrm{s}$ \\
\hline Radial velocity & $0 \mathrm{~m} / \mathrm{s}$ \\
\hline Gauge pressure & $0 \mathrm{~Pa}$ \\
\hline Number of iteratiơns & 10,000 \\
\hline
\end{tabular}


Table B.2: FLUENT settings, tailpipe geometry.

\begin{tabular}{|c|c|}
\hline Geometry & \\
\hline Domain size: H x W & $1.2 \mathrm{~m} \mathrm{x} \mathrm{10} \mathrm{m}$ \\
Tailpipe radius & $20 \mathrm{~mm}$ \\
Tailpipe wall thickness & $1 \mathrm{~mm}$ \\
Tailpipe length & $500 \mathrm{~mm}$ \\
\hline Mesh Details & \\
\hline Number of mesh elements & 415,094 \\
\hline FLUENT settings & $102 \mathrm{~m} / \mathrm{s}$ \\
\hline Axial inlet velocity in pipe & $3.34 \%$ \\
Turbulent intensity & $40 \mathrm{~mm}($ following $[31]$ ) \\
Hydraulic diameter (turbulence property) & \\
No slip wall conditions for all pipe surfaces & \\
Initialization values: & from pipe inlet \\
Turbulent properties & $10 \mathrm{~m} / \mathrm{s}$ \\
Axial velocity & 12,000 \\
Number of iterations & \\
\hline
\end{tabular}




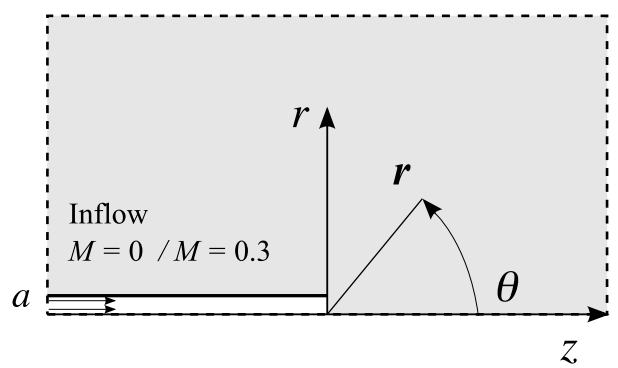

Figure B.1: Studied axisymmetric configuration for a medium with and without jet as inflow conditions in the pipe. 


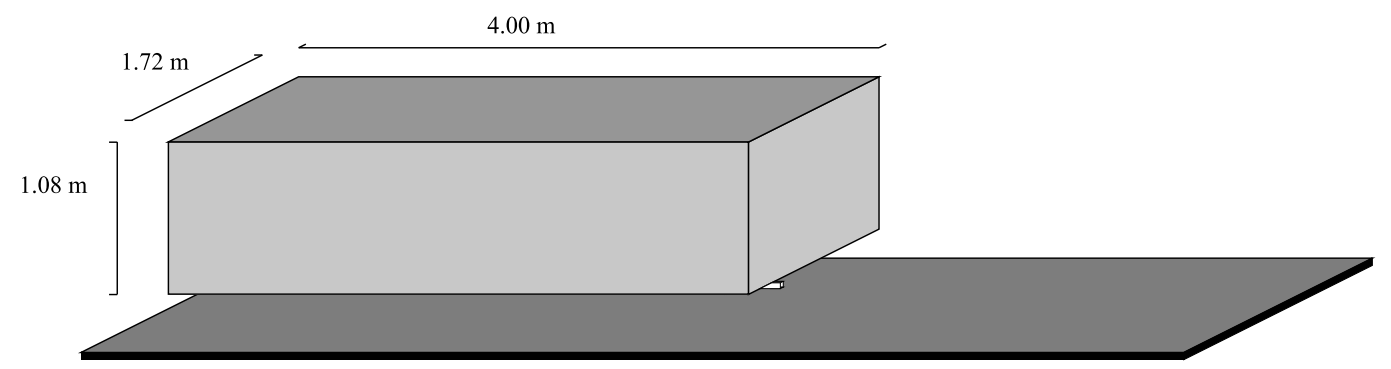

a)
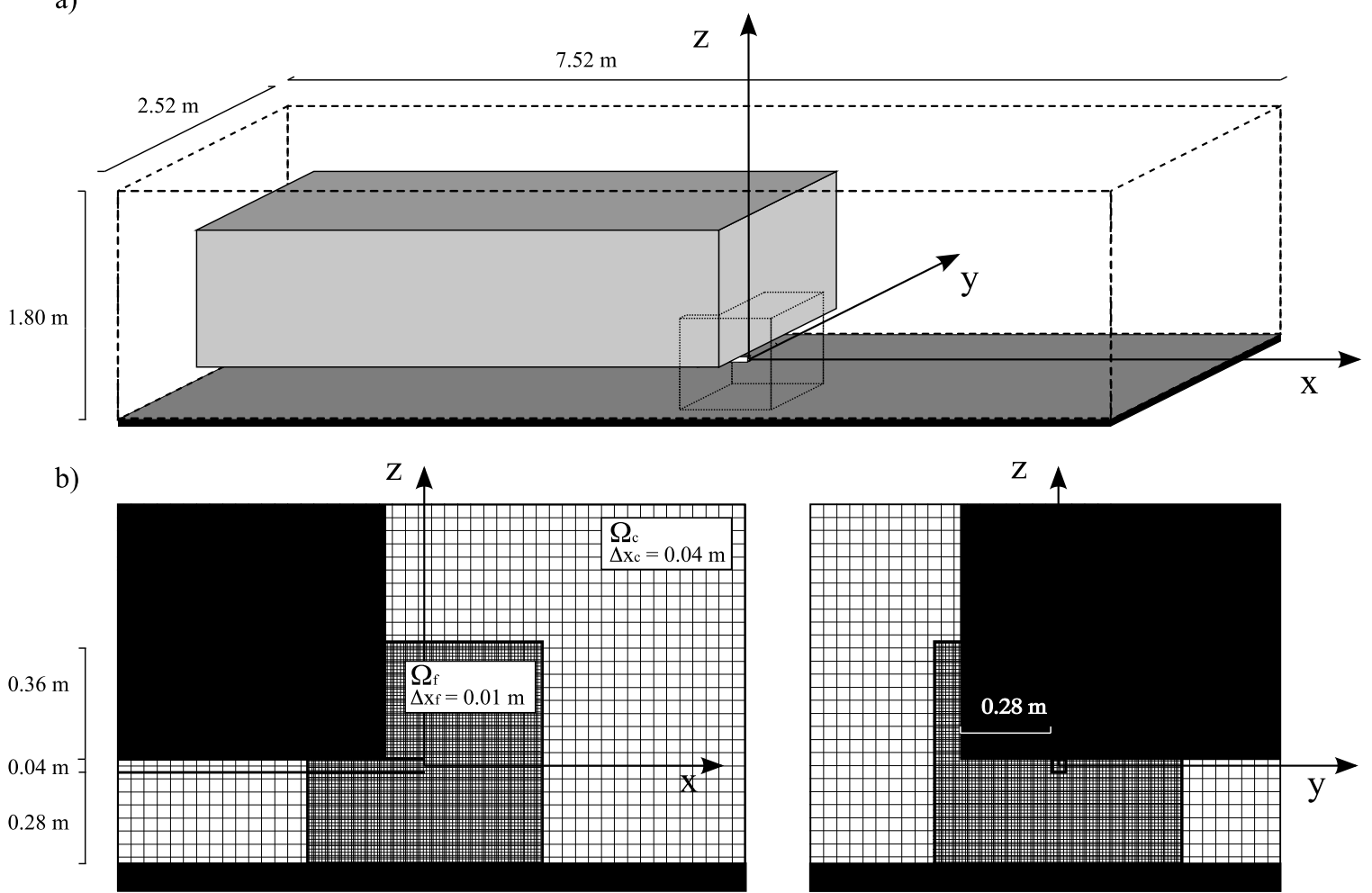

c) $y=0 \mathrm{~m}$

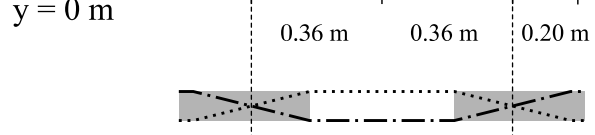

Data transfer coarse to fine grid

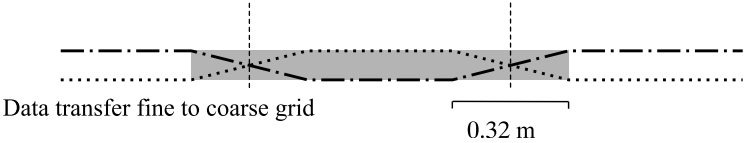

Z

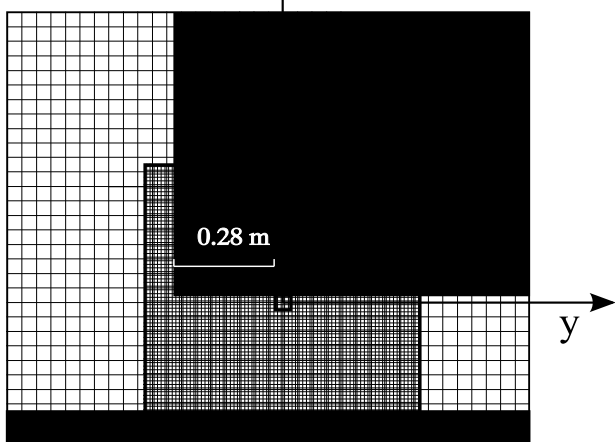

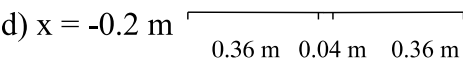

Data transfer region

... Multiplication window fine grid values

-.- Multiplication window coarse grid values

Figure B.2: a) Studied configuration of an exhaust pipe over a rigid ground surface in the presence of a rigid body; b) Discretization volumes of the multi-domain extended Fourier PSTD method covering the computational domain: the small volume contains the fine PSTD mesh and the large volume contains the coarse PSTD mesh. Receiver positions are located outside the largest volume and computed by integrating the solution at the outer surfaces of the larger volume; c) Part of the cross section at $y=0 \mathrm{~m}$ with coarse and fine grids illustrated. Data transfer methodology is depicted below the cross section. d) Part of the cross section at $x=-0.2 \mathrm{~m}$. 


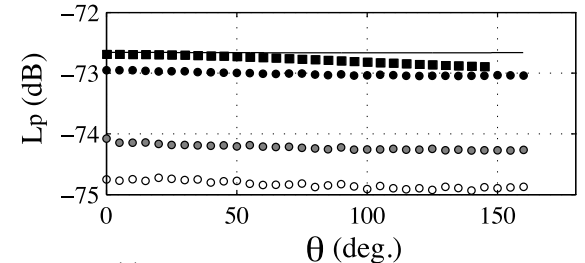

(a)

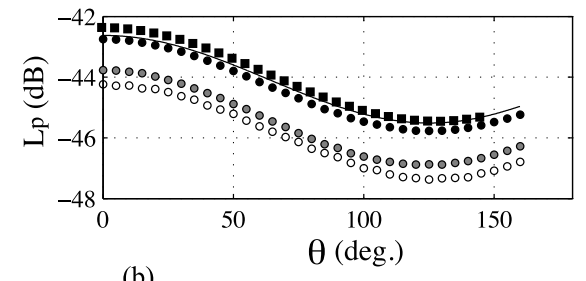

(b)

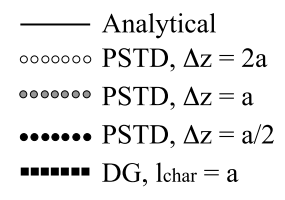

Figure B.3: Directivity $L_{p}(\mathrm{~dB})$ for the axisymmetric configuration of Fig. B.1 for (a) $63 \mathrm{~Hz}$ and (b) $2000 \mathrm{~Hz}$, and the absence of a jet flow as a function of the grid discretization in PSTD.
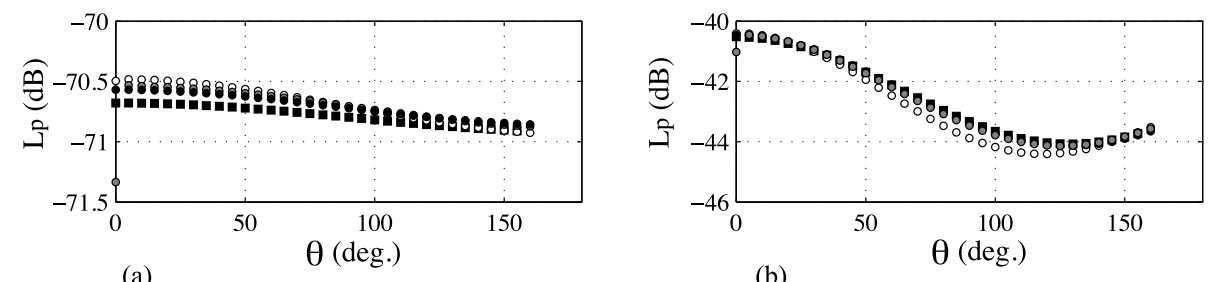

0000000 PSTD, $\Delta \mathrm{z}=2 \mathrm{a}$

0000000 PSTD, $\Delta z=\mathrm{a}$

..... PSTD,$\Delta z=\mathrm{a} / 2$

-.m. DG, 1 char $=\mathrm{a}$

(b)

Figure B.4: Directivity $L_{p}(\mathrm{~dB})$ for the 3D free field configuration of Fig. B.1(b) for (a) $63 \mathrm{~Hz}$ and (b) $2000 \mathrm{~Hz}$, and the absence of a jet flow as a function of the grid discretization in PSTD. 


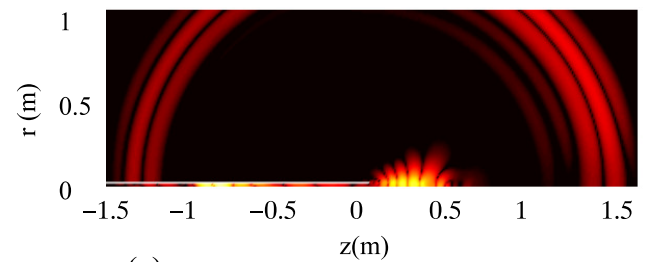

(a)

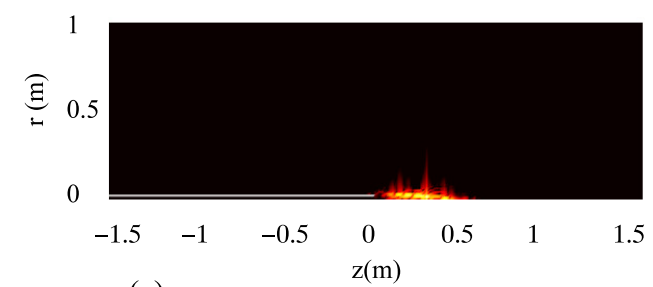

(c)

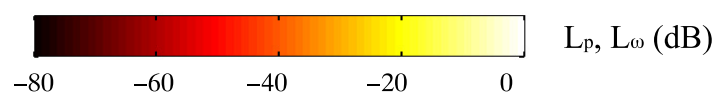

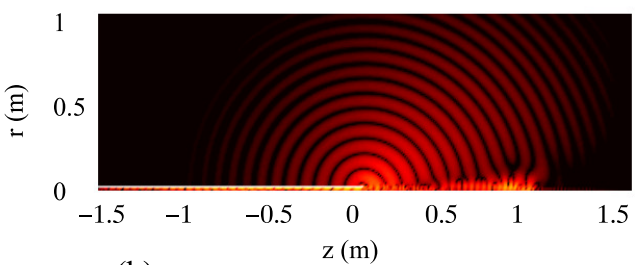

(b)

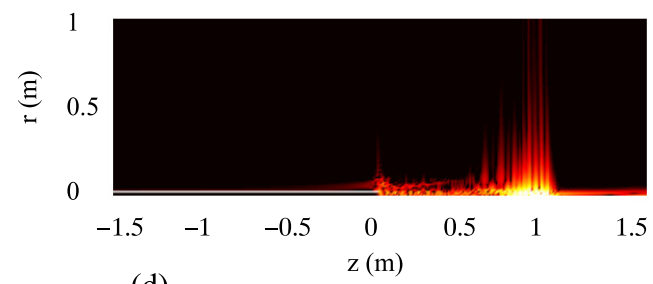

(d)

Figure B.5: LEE solved by PSTD for configuration of Fig. B.1. Snapshots of a part of the domain of the instantaneous pressure level $L_{p}(t)=20 \log _{10}\left(|p(t)| / 1 \mathrm{e}^{-5}\right)$ and vorticity level $L_{\omega}(t)=$ $20 \log _{10}\left(|\omega(t)| / 1 \mathrm{e}^{-5}\right)$ at two time instances. (a) $L_{p}$ at $t=0.007 \mathrm{~s}$, (b) $L_{p}$ at $t=0.018 \mathrm{~s}$, (c) $L_{\omega}$ at $t=0.007 \mathrm{~s},(\mathrm{~d}) L_{\omega}$ at $t=0.018 \mathrm{~s}$.

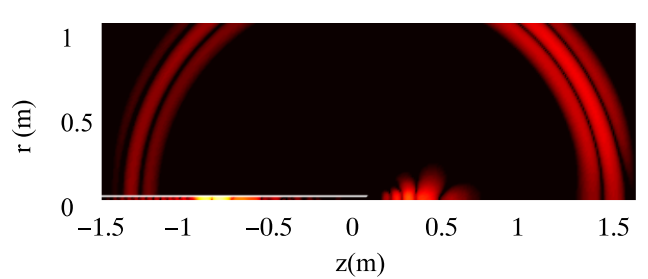

(a)

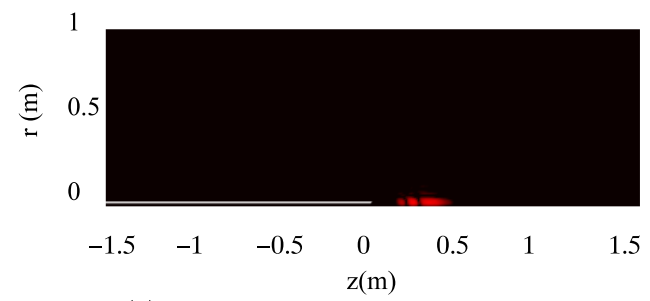

(c)

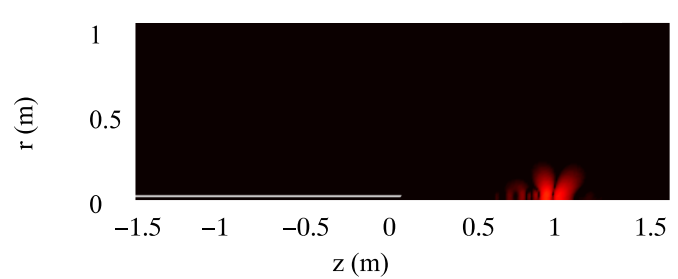

(b)

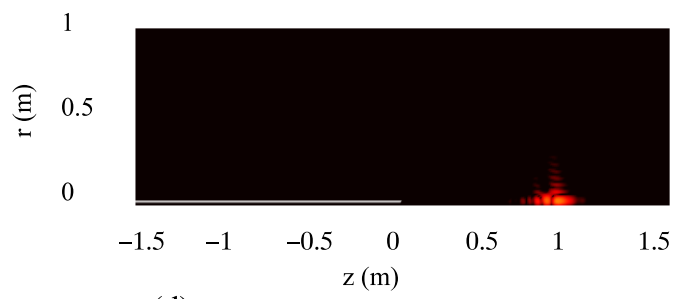

(d)

Figure B.6: LEE solved by PSTD for configuration of Fig. B.1 with filter with $k_{c}=\frac{\pi}{2 a} \mathrm{~m}^{-1}$. Snapshots of a part of the domain of the instantaneous pressure level $L_{p}(t)=20 \log _{10}\left(|p(t)| / 1 \mathrm{e}^{-5}\right)$ and vorticity level $L_{\omega}(t)=20 \log _{10}\left(|\omega(t)| / 1 \mathrm{e}^{-5}\right)$ g t 4 two time instances. Colorbar as in Fig. B.5. (a) $L_{p}$ at $t=0.007 \mathrm{~s}$, (b) $L_{p}$ at $t=0.018 \mathrm{~s},\left(\right.$ c) $L_{\omega}$ at $t=0.007 \mathrm{~s}$, (d) $L_{\omega}$ at $t=0.018 \mathrm{~s}$. 


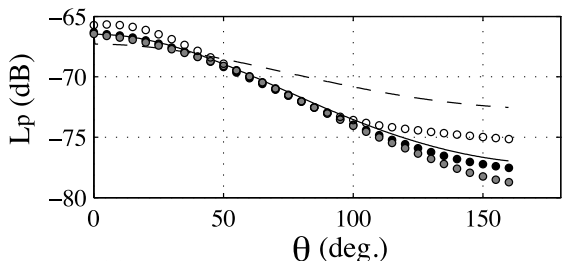

(a)

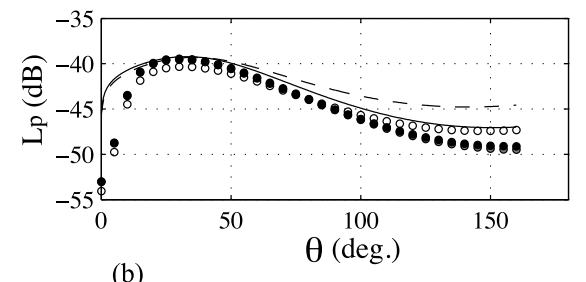

(b)

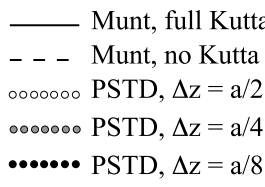

$\cdots$ PSTD,$\Delta z=\mathrm{a} / 8$

Figure B.7: Directivity $L_{p}(\theta)(\mathrm{dB})$ for configuration of Fig. B.1 for (a) $63 \mathrm{~Hz}$ and (b) $2000 \mathrm{~Hz}$, with jet flow as a function of the grid discretization in PSTD, $k_{c}=\pi / a$ and $m=8$.

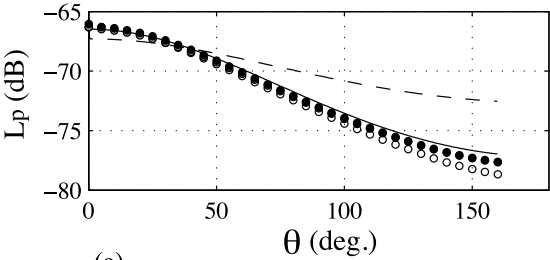

(a)

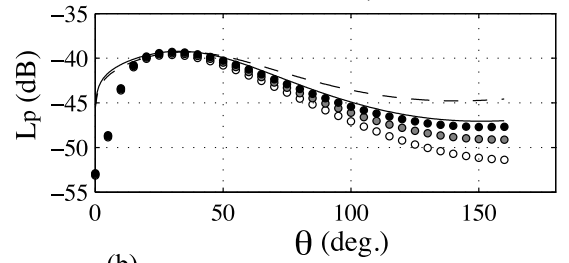

(b)

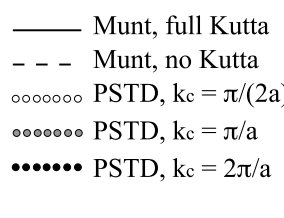

- Munt, full Kutta 0000000 PSTD, $\mathrm{kc}_{\mathrm{c}}=\pi /(2 \mathrm{a})$ 0000000 PSTD, $\mathrm{kc}_{\mathrm{c}}=\pi / \mathrm{a}$

Figure B.8: Directivity $L_{p}(\theta)(\mathrm{dB})$ for configuration of Fig. B.1 with jet flow, for (a) $63 \mathrm{~Hz}$ and (b) $2000 \mathrm{~Hz}$, as a function of cut-off wavenumber $k_{c}$ of filtering acoustic variables in PSTD, $\Delta z_{c}=a / 2$, $\Delta z_{f}=a / 8$ and $m=8$.

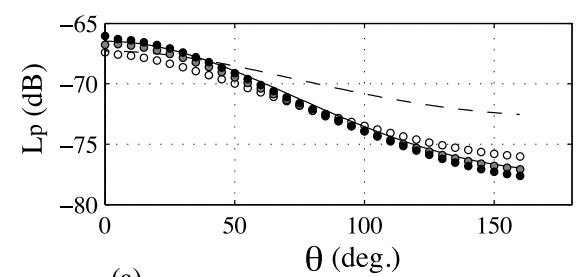

(a)

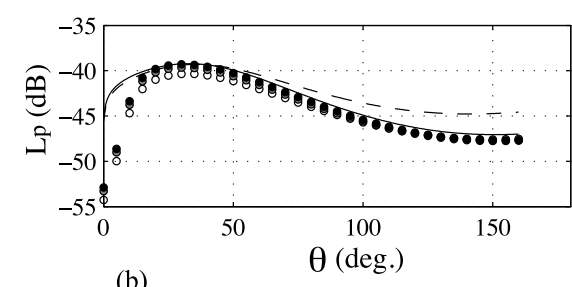

(b)
— Munt, full Kutta _ - - Munt, no Kutta 0000000 PSTD, $\mathrm{mf}=2$

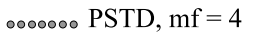
•.... PSTD, $\mathrm{mf}=8$

Figure B.9: Directivity $L_{p}(\theta)(\mathrm{dB})$ for configuration of Fig. B.1 with jet flow, for (a) $63 \mathrm{~Hz}$ and (b) $2000 \mathrm{~Hz}$, as a function of mean flow filter coefficient $m$ in PSTD, $\Delta z_{c}=a / 2, \Delta z_{f}=a / 8$ and $k_{c}=2 \pi / a$. 

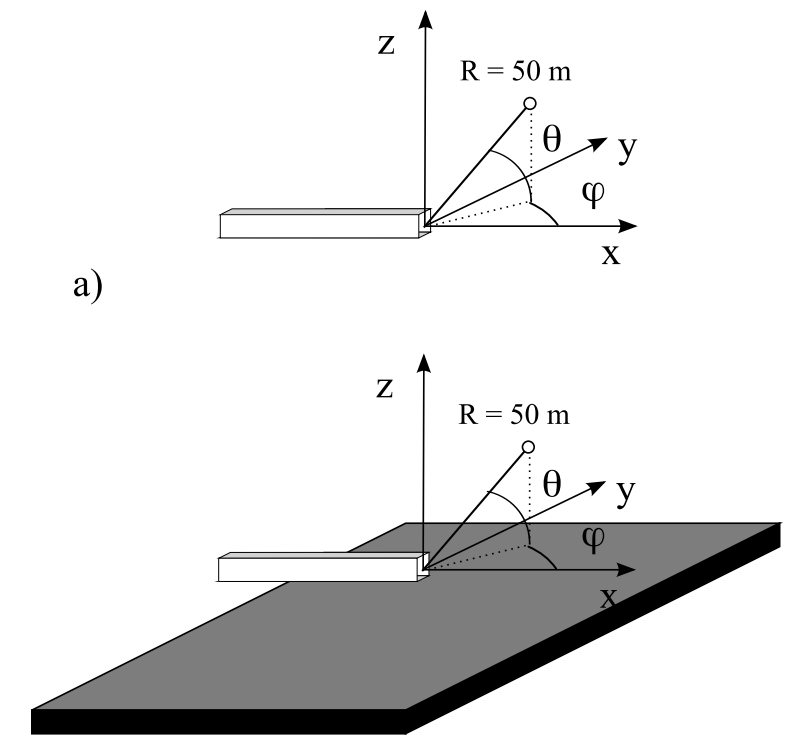

b)

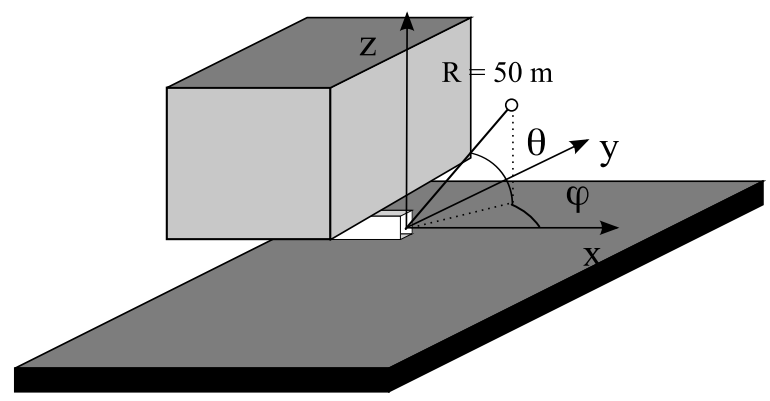

c)

Figure B.10: Schematic representation of the studied configurations. Dimensions can be found from Fig. B.2. a) Exhaust pipe in free field, b) Exhaust pipe over a rigid ground surface, c) Exhaust pipe over a rigid ground surface in the presence of a rigid body. 


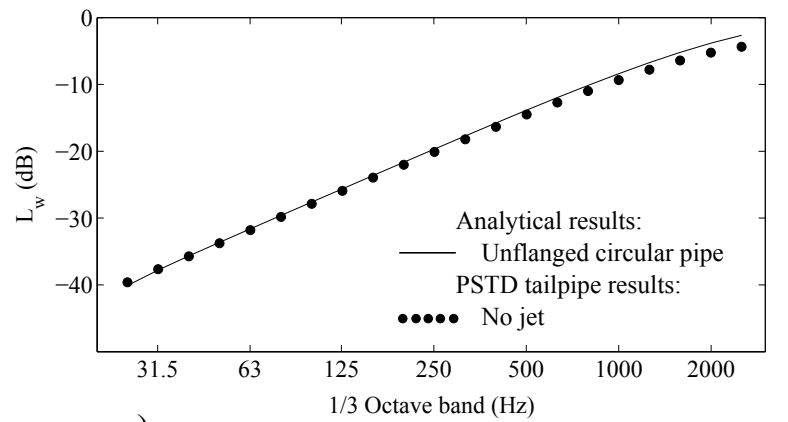

a)

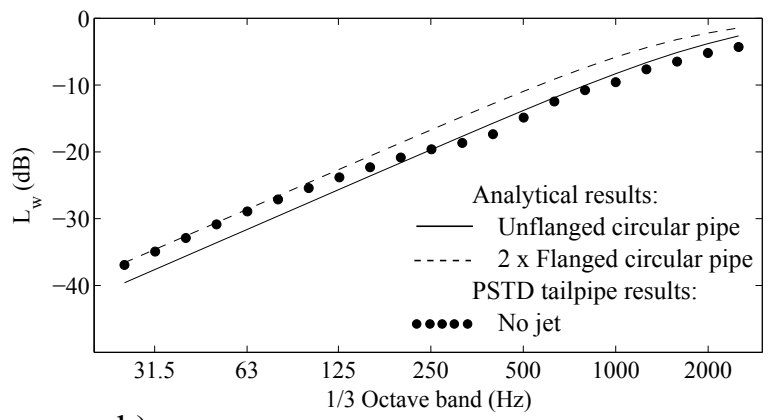

b)

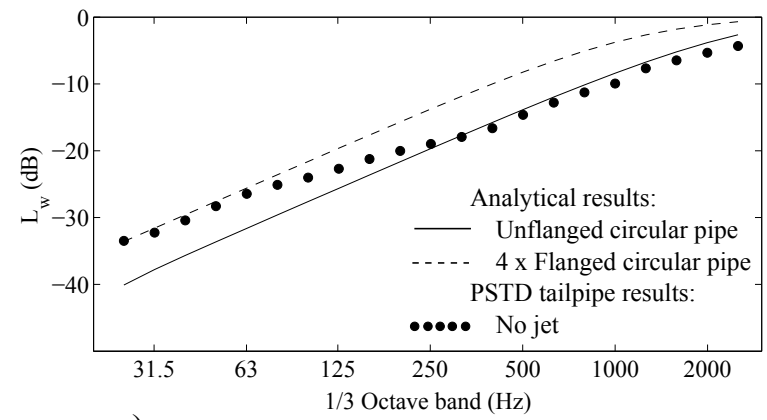

c)

Figure B.11: Sound power level $L_{w}$ relative to the incident power in the exhaust pipe. a) Exhaust pipe in free field, b) Exhaust pipe over a rigid ground surface, c) Exhaust pipe over a rigid ground surface in the presence of a rigid body. 


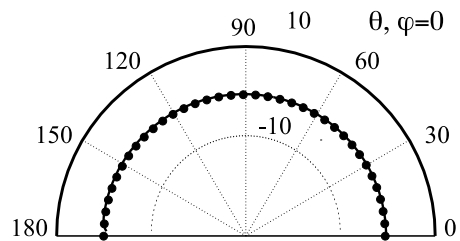

a) $\mathrm{L}_{\mathrm{p}}, 63 \mathrm{~Hz}$

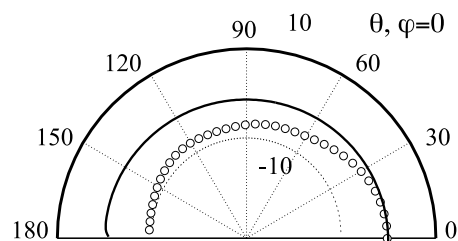

c) $\mathrm{L}_{\mathrm{p}}, 63 \mathrm{~Hz}$

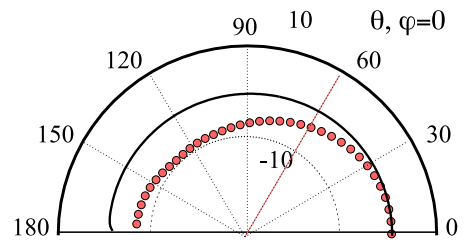

e) $\mathrm{L}_{p}, 63 \mathrm{~Hz}$

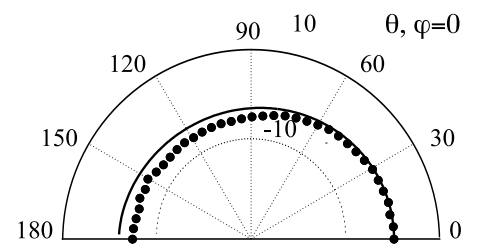

b) $\mathrm{Lp}, 2000 \mathrm{~Hz}$

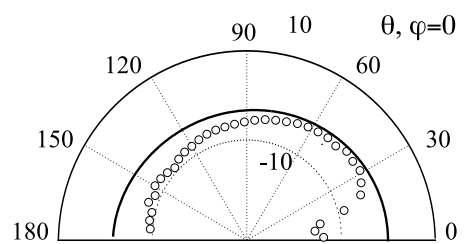

d) $\mathrm{L}_{p}, 2000 \mathrm{~Hz}$

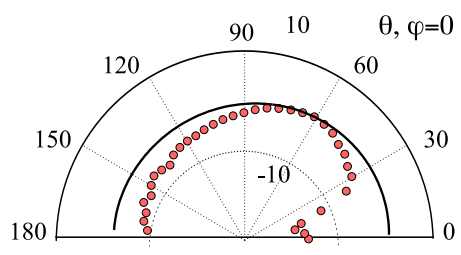

f) $\mathrm{Lp}_{\mathrm{p}}, 2000 \mathrm{~Hz}$
Analytical results:

Unflanged circular pipe, no jet PSTD tailpipe results:

..... No jet

Analytical results:

Unflanged circular pipe, no jet PSTD tailpipe results:

00000 Cold jet, $M=0.3, \mathrm{~T}_{0}=293 \mathrm{~K}$

Analytical results:

Unflanged circular pipe, no jet PSTD tailpipe results:

๑000० Hot Jet, $\mathrm{M}=0.19, \mathrm{~T}_{0}=723 \mathrm{~K}$

Figure B.12: Exhaust pipe in free field, directivity plots in the $z=0$ plane, (a,b) No jet, (c,d) Cold jet, $(\mathrm{e}, \mathrm{f})$ Hot jet. 

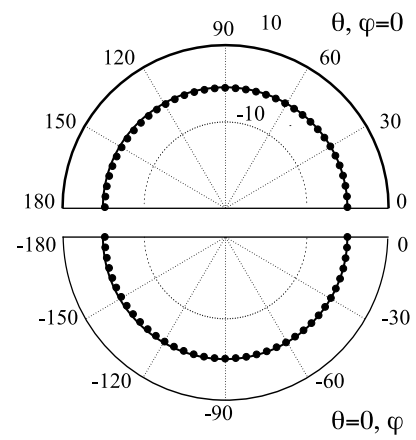

a) $\mathrm{L}_{\mathrm{p}}, 63 \mathrm{~Hz}$
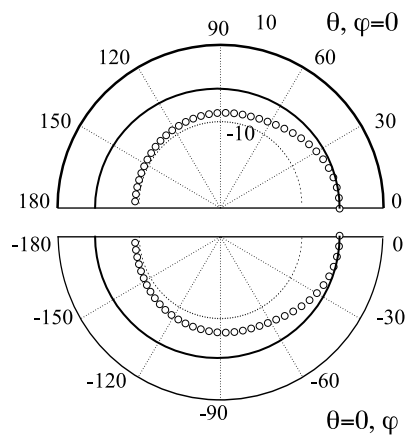

c) $\mathrm{Lp}_{\mathrm{p}}, 63 \mathrm{~Hz}$
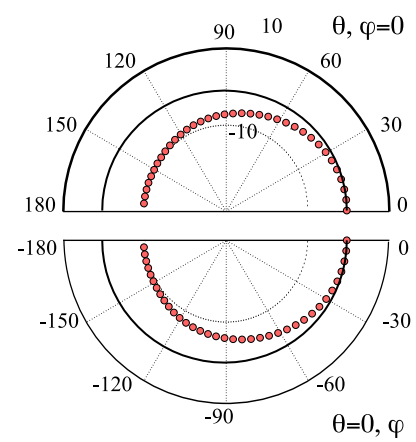

e) $\mathrm{Lp}_{\mathrm{p}}, 63 \mathrm{~Hz}$
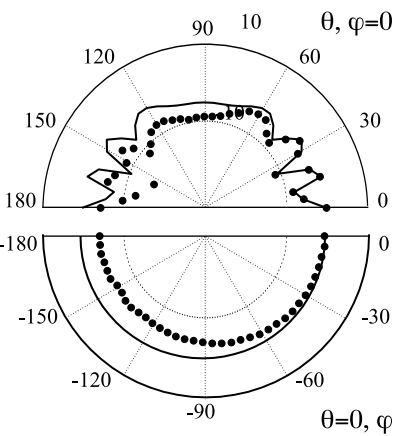

b) $\mathrm{L}_{\mathrm{p}}, 2000 \mathrm{~Hz}$

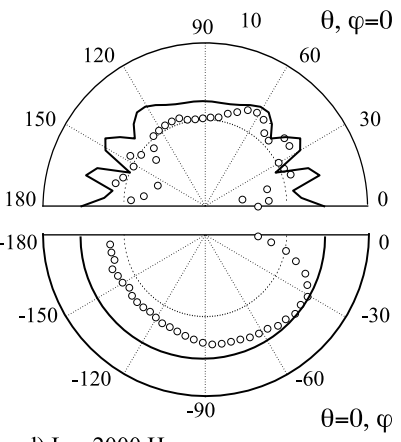

d) $\mathrm{Lp}_{\mathrm{p}}, 2000 \mathrm{~Hz}$
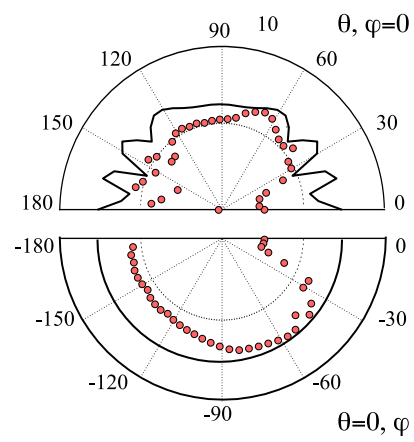

f) $\mathrm{Lp}_{\mathrm{p}}, 2000 \mathrm{~Hz}$
Analytical results:

- Point source above rigid ground, no jet PSTD tailpipe results:

•.... No jet

\footnotetext{
Analytical results:

— Point source above rigid ground, no jet PSTD tailpipe results: 00000 Cold jet, $\mathrm{M}=0.3, \mathrm{~T}_{0}=293 \mathrm{~K}$
}

Figure B.13: Exhaust pipe over a rigid ground surface, directivity plots in the $z=0$ and $y=0$ plane, $(\mathrm{a}, \mathrm{b})$ No jet, (c,d) Cold jet, (e,f) Hot jet. 

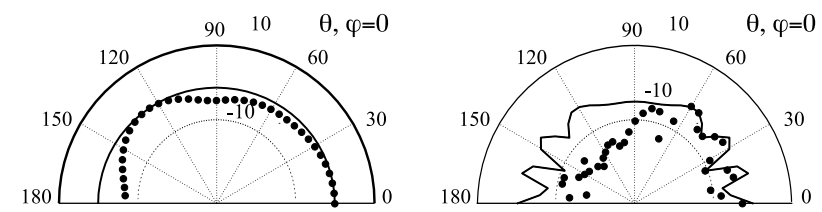
Analytical results:
Point source above rigid ground, no jet PSTD tailpipe results:
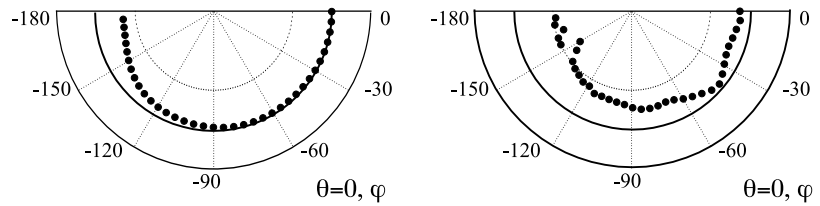

•... No jet

a) $\mathrm{Lp}_{\mathrm{p}}, 63 \mathrm{~Hz}$

b) $\mathrm{L}_{p}, 2000 \mathrm{~Hz}$
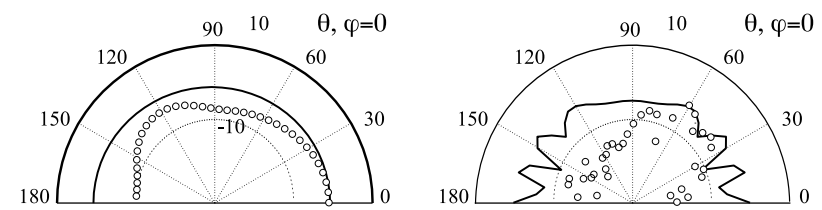
Analytical results:
Point source above rigid ground, no jet
PSTD tailpipe results:
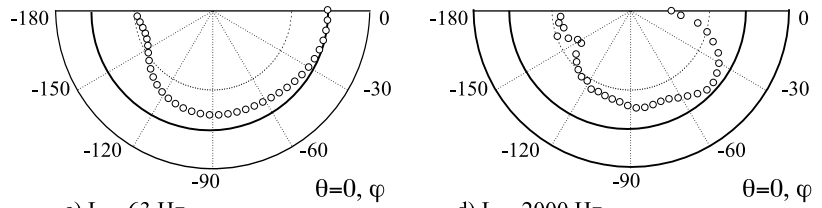

00000 Cold jet, $\mathrm{M}=0.3, \mathrm{~T}_{0}=293 \mathrm{~K}$

c) $\mathrm{Lp}_{\mathrm{p}}, 63 \mathrm{~Hz}$
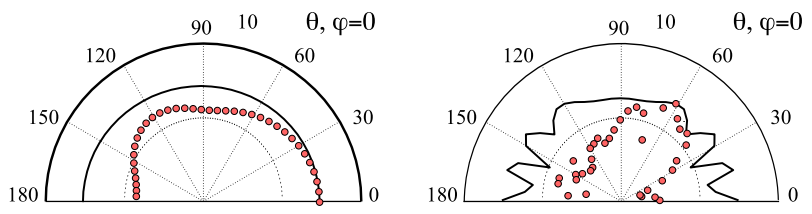

Analytical results:

Point source above rigid ground, no jet PSTD tailpipe results:

๑०००० Hot jet, $\mathrm{M}=0.19, \mathrm{~T}_{0}=723 \mathrm{~K}$

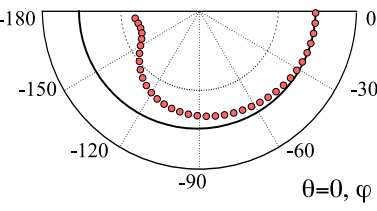

e) $\mathrm{Lp}_{\mathrm{p}}, 63 \mathrm{~Hz}$

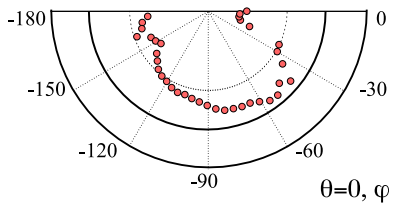

f) $\mathrm{Lp}_{\mathrm{p}}, 2000 \mathrm{~Hz}$

Figure B.14: Exhaust pipe over a rigid ground surface in the presence of a rigid body, directivity plots in the $z=0$ and $y=0$ plane, (a,b) No jet, (c,d) Cold jet, (e,f) Hot jet. 


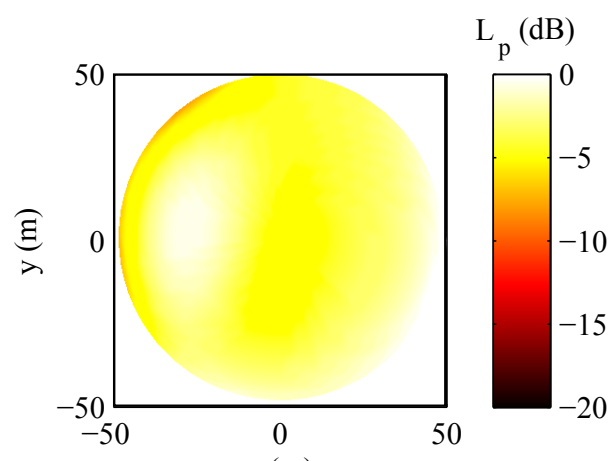

a) No Jet, $63 \mathrm{~Hz}$

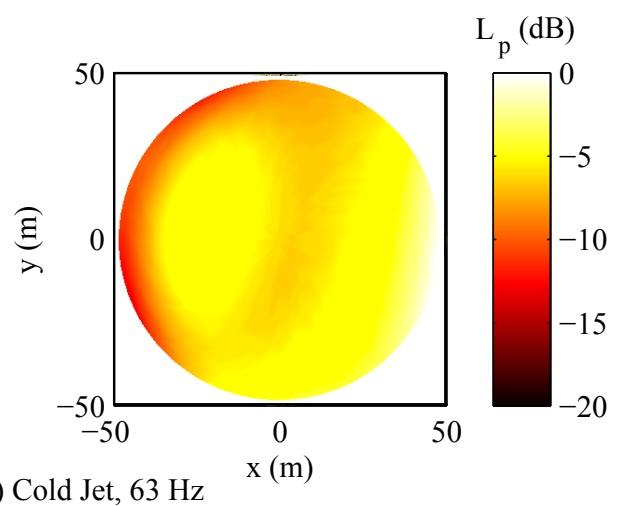

a) Cold Jet, $63 \mathrm{~Hz}$

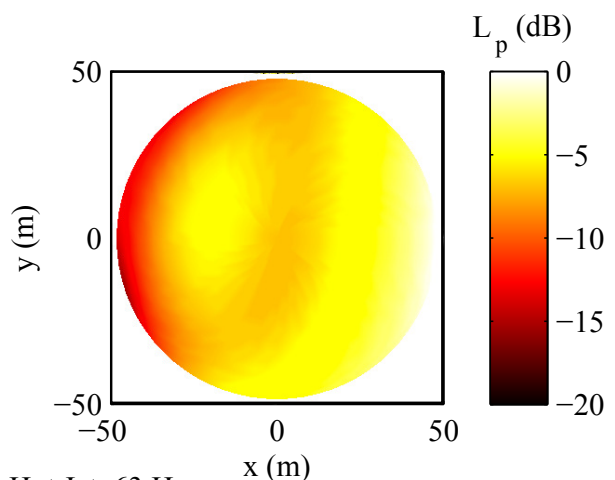

a) Hot Jet, $63 \mathrm{~Hz}$

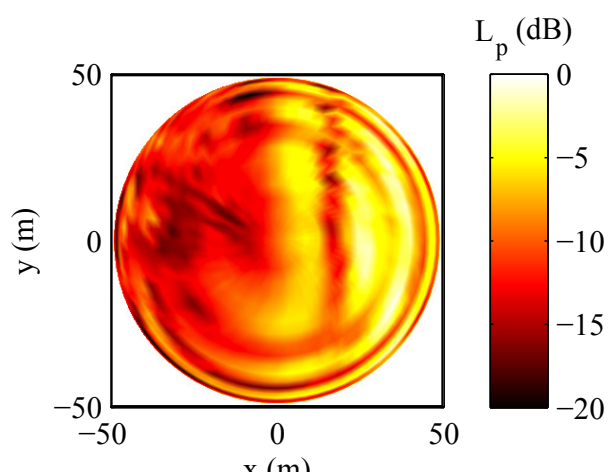

b) No Jet, $2000 \mathrm{~Hz}$
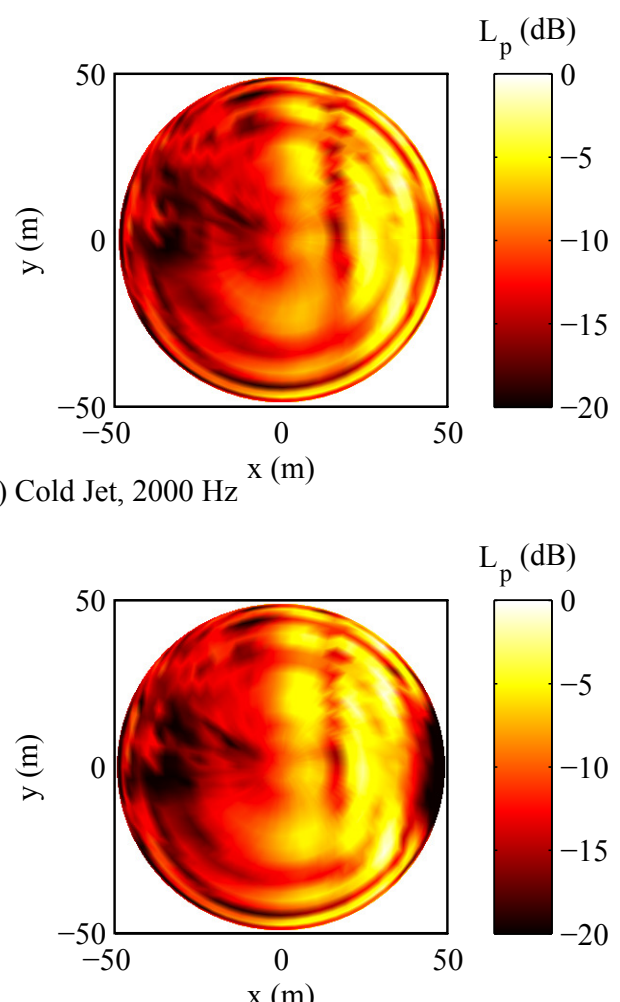

b) Hot Jet, $2000 \mathrm{~Hz}$ X(m)

Figure B.15: Top view of $L_{p}$ in a hemisphere with $R=50 \mathrm{~m}$ in the $63 \mathrm{~Hz}$ (left) and $2000 \mathrm{~Hz}$ (right) $1 / 3$ octave band for the configuration with tailpipe, ground surface and body. (top) No jet, (middle) Cold jet, (bottom) Hot jet. 

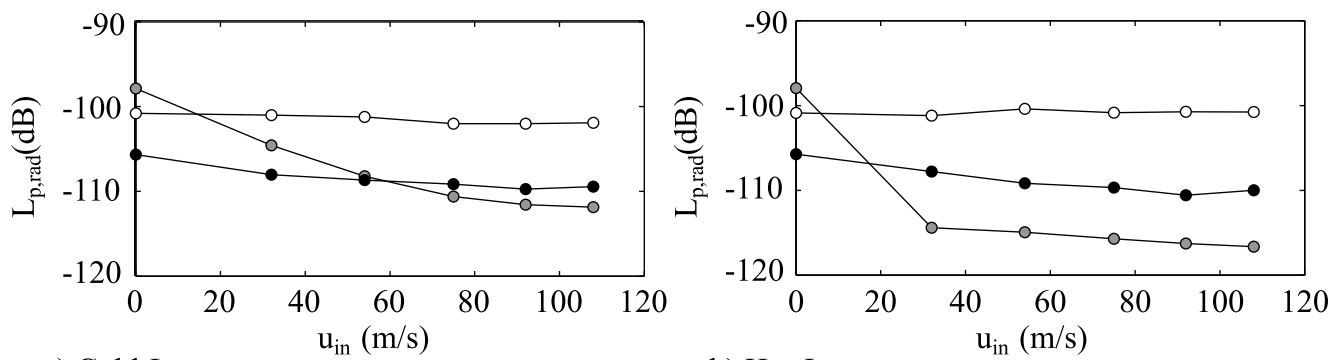

a) Cold Jet

b) Hot Jet

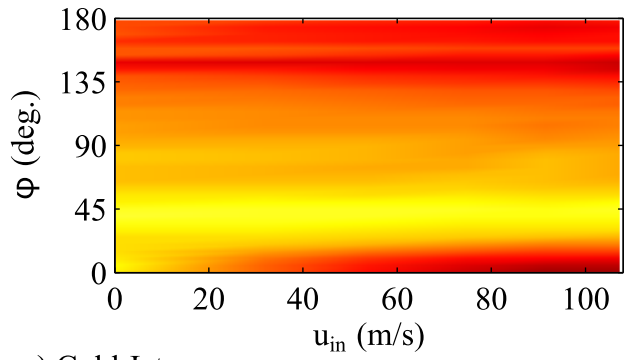

c) Cold Jet

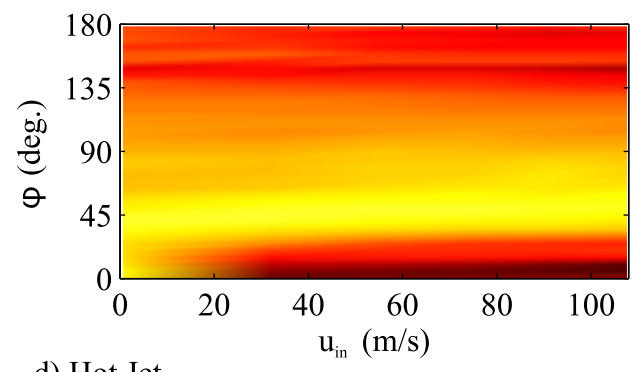

d) Hot Jet

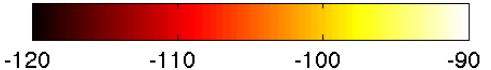

Figure B.16: Radiated noise levels $L_{p, r a d}$ for various inflow velocities and inlet temperatures (see text) for $\theta=0$ deg. Grey circles: $\phi=-5$ deg., white circles: $\phi=-90$ deg., black circles: $\phi=-175$ deg. (a,c) Cold jet, (b,d) Hot jet. 


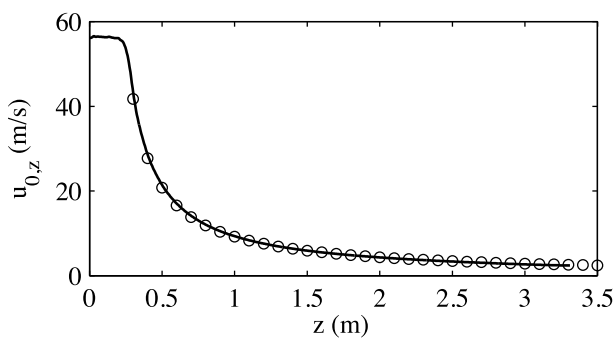

(a)

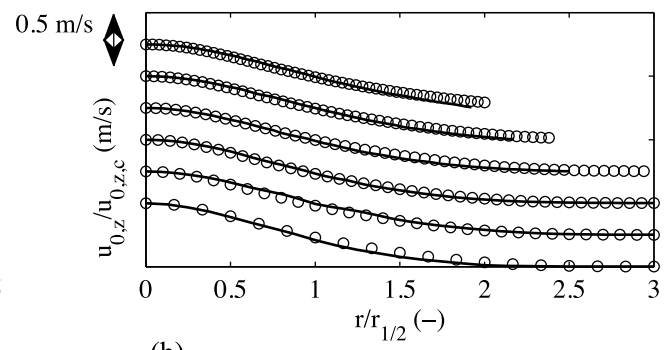

(b)
0000000 SHL30

RANS

Figure B.17: (a) Axial velocity component at $\mathrm{r}=0$ from RANS results and the SHL30 measurements [29]; (b) Axial velocity component normalized to the centerline velocity $u_{0, z, c}$ and plotted to the normalized radial distance for $\mathrm{z}=0.8 \mathrm{~m}, 1.2 \mathrm{~m}, 1.6 \mathrm{~m}, 2.0 \mathrm{~m}, 2.4 \mathrm{~m}$ and $\mathrm{z}=2.8 \mathrm{~m}$.

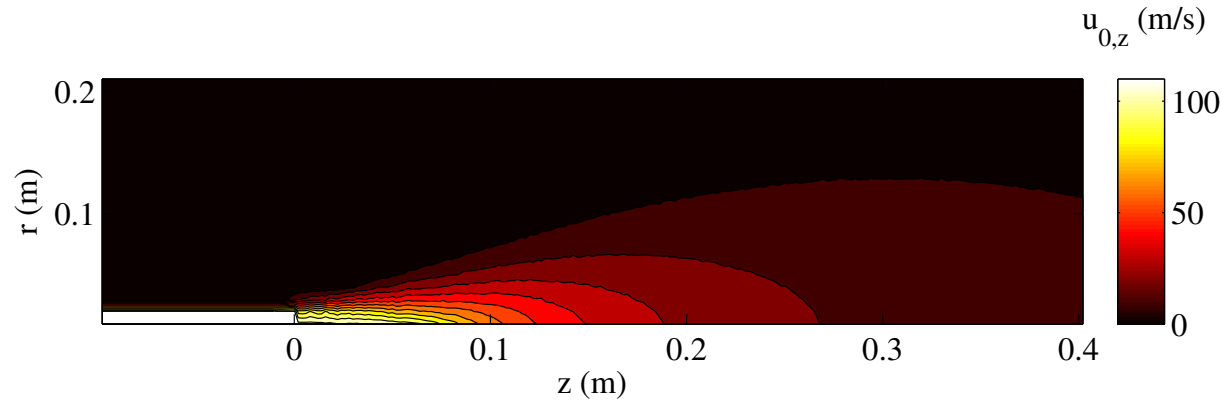

Figure B.18: Mean axial velocity component $u_{0, z}$ calculated by RANS and filtered by Eq. (5) with $m=2$. 